\title{
MORITA THEORY FOR FINITARY 2-CATEGORIES
}

\author{
VOLODYMYR MAZORCHUK AND VANESSA MIEMIETZ
}

\begin{abstract}
We develop Morita theory for finitary additive 2-representations of finitary 2-categories. As an application we describe Morita equivalence classes for 2-categories of projective functors associated to finite dimensional algebras and for 2-categories of Soergel bimodules.
\end{abstract}

\section{INTRODUCTION}

Classical Morita theory (see $[\mathrm{Mo}]$ ) describes when two rings have equivalent categories of representations. By now there are many generalizations of this theory by varying what is represented and where it is represented (see e.g. [Ri, Ke, Kn, BV, BD, To]).

In [MM1, MM2, MM3], motivated by recent success of higher categorical methods in both topology (see e.g. [Kh, St]) and representation theory (see e.g. [Ar, Gr, LLT, CR]), we started a systematic study of the 2-representation theory of finitary 2-categories. The latter should be thought of as 2-analogues of finite dimensional algebras. Assuming the existence of adjunction morphisms (which should be thought of as 2-analogues of an involution on a finite dimensional algebra) we constructed certain natural classes of 2-representations and established, under some natural conditions, a 2-analogue of Schur's lemma as well as analogues of other criteria for simplicity of a representation. In this article we drop the assumption on existence of adjunction morphisms and study Morita theory for arbitrary finitary 2 -categories.

In view of the different known versions of Morita theory (as in [Ri, Ke, Kn, BV, $\mathrm{BD}, \mathrm{To}]$ ), our main results are as expected. Each finitary 2-category is biequivalent to the (opposite of the) endomorphism 2-category of its representable (principal) 2-representations. Our main result asserts that Morita equivalent finitary 2-categories can be obtained one from the other by taking the (opposite of the) endomorphism category of a suitable "projective generator" or, in other words, by adding and/or removing retracts of principal 2-representations. Along the way we obtain a full classification of all retracts of principal 2-representations and show that indecomposable retracts are given by indecomposable 1-morphisms which square to an idempotent (the latter might decompose). In line with the common phenomenon that "useful" categorifications of semisimple algebras and categories are usually not semisimple (see e.g. [CR, St]), retracts of projective 2-representations do not necessarily split off as direct summands.

As an application we describe Morita equivalence classes for finitary 2-categories of projective functors associated to finite dimensional algebras. This is an important class of finitary 2-categories which originally appeared in [MM1], and which later, in [MM3], played the role of an important prototype of a certain class of "simple" 
finitary 2-categories appearing in an analogue of the Artin-Wedderburn Theorem. As it turns out, the classification is not obvious and Morita equivalence classes are described in terms of adding/removing semi-simple direct summands (under some additional restrictions). We also show that for 2-categories of Soergel bimodules the Morita equivalence classes correspond to isomorphism classes of Coxeter systems.

The paper is organized as follows: In Section 2 we recall the classical Morita theory for finitary $\mathbb{k}$-linear categories. Section 3 is a brief introduction to the 2-representation theory of finitary 2-categories. In Section 4 we define and classify projective 2-representations. Although our main techniques come from category theory (see $[\mathrm{BD}]$ ), we also use the classical combinatorial description of idempotent matrices with non-negative integer coefficients from [Fl]. In Section 5 we prove our main result. We complete the paper with some examples and applications in Section 6 .

Acknowledgment. A substantial part of the paper was written during a visit of the second author to Uppsala University, whose hospitality is gratefully acknowledged. The visit was supported by the Swedish Research Council. The first author is partially supported by the Swedish Research Council and the Royal Swedish Academy of Sciences. The second author is partially supported by ERC grant PERG07-GA-2010-268109 and by EPSRC grant EP/K011782/1. We are really grateful to the referees for very careful reading of the paper, for pointing out several subtle inaccuracies in the original version and for many very helpful suggestions.

\section{Morita THEORY FOR FINITARY $\mathbb{k}$-LINEAR CATEGORIES}

In this section we present some well-known results from classical Morita theory for finite dimensional associative algebras in a form that resembles our subsequent treatment of Morita theory for finitary 2-categories. We denote by $\mathbb{N}$ the set of positive integers.

2.1. Finitary $\mathbb{k}$-linear categories. Throughout the article, let $\mathbb{k}$ be an algebraically closed field. A $\mathbb{k}$-linear category is a category enriched over the category $\mathbb{k}$-Mod of $\mathbb{k}$-vector spaces, meaning that morphisms form $\mathbb{k}$-vector spaces and composition of morphisms is $\mathbb{k}$-bilinear. A $\mathbb{k}$-linear category $\mathcal{C}$ is called finitary provided that

- $\mathcal{C}$ is skeletally finite, that is it has finitely many isomorphism classes of objects;

- all morphism spaces in $\mathcal{C}$ are finite dimensional (over $\mathbb{k}$ ).

A finitary $\mathbb{k}$-linear category $\mathcal{C}$ is called reduced if we additionally have

- for any $i \in \mathcal{C}$ the (finite dimensional unital) $\mathbb{k}$-algebra $\mathcal{C}(i, i)$ is local.

2.2. Representations of finitary $\mathbb{k}$-linear categories. Let $\mathcal{C}$ be a finitary $\mathbb{k}$-linear category. By a representation of $\mathcal{C}$ we mean a $\mathbb{k}$-linear functor $\mathrm{M}: \mathcal{C} \rightarrow \mathbb{k}$-Mod. A finitary representation of $\mathcal{C}$ is a $\mathbb{k}$-linear functor $\mathrm{M}: \mathcal{C} \rightarrow \mathbb{k}$-mod, where $\mathbb{k}$-mod 
denotes the category of finite-dimensional $\mathbb{k}$-vector spaces. Given two representations $\mathrm{M}$ and $\mathrm{N}$ of $\mathcal{C}$, a morphism $\alpha: \mathrm{M} \rightarrow \mathrm{N}$ is just a natural transformation from $\mathrm{M}$ to $\mathrm{N}$. All (finitary) representations of $\mathcal{C}$ together with morphisms between them form an abelian category denoted $\mathcal{C}$-Mod (resp. $\mathcal{C}$-mod).

One important example of a representation of $\mathcal{C}$ is given by the representable functor $\mathcal{C}\left(i,{ }_{-}\right)$for $i \in \mathcal{C}$. For $\mathrm{M} \in \mathcal{C}$-Mod we have the Yoneda isomorphism

$$
\operatorname{Hom}_{\mathcal{C}}\left(\mathcal{C}\left(i,{ }_{-}\right), M\right) \cong M(i)
$$

given by $\varphi \mapsto \varphi\left(\mathbb{1}_{i}\right)$. In particular, as $\mathrm{M} \mapsto \mathrm{M}(\mathrm{i})$ is exact, it follows that the representation $\mathcal{C}\left(i,{ }_{-}\right)$is projective. We denote by $\mathcal{C}$-proj the full additive subcategory of $\mathcal{C}$-mod consisting of all projective $\mathcal{C}$-modules and note that $\mathcal{C}$-proj coincides with the full additive closure (by which we mean closure under direct sums, direct summands, and isomorphisms) of the $\mathcal{C}\left(i,{ }_{-}\right)$for $i \in \mathcal{C}$. Further, any indecomposable projective $\mathcal{C}$-module is isomorphic to $\mathcal{C}\left(i,{ }_{-}\right) \cdot e$, where $e$ is an indecomposable idempotent in $\mathcal{C}(i, i)$ (the latter being canonically isomorphic to $\left.\operatorname{End}_{\mathcal{C}}\left(\mathcal{C}\left(i,{ }_{-}\right)\right)^{\text {op }}\right)$.

A progenerator for $\mathcal{C}$-mod is a full subcategory $\mathcal{P}$ in $\mathcal{C}$-proj such that the additive closure of $\mathcal{P}$ coincides with $\mathcal{C}$-proj.

2.3. Morita theorem. For reduced $\mathbb{k}$-linear categories the Morita theorem looks as follows:

Proposition 1. Let $\mathcal{A}$ and $\mathcal{C}$ be two reduced finitary $\mathbb{k}$-linear categories. Then the categories $\mathcal{A}$-mod and $\mathcal{C}$-mod are equivalent if and only if the categories $\mathcal{A}$ and $\mathcal{C}$ are equivalent.

Proof. Let $\Phi: \mathcal{A} \rightarrow \mathcal{C}$ be an equivalence with inverse $\Psi: \mathcal{C} \rightarrow \mathcal{A}$. These functors give rise to functors

$$
\text { - } \circ \Phi: \mathcal{C} \text {-mod } \rightarrow \mathcal{A} \text {-mod } \quad \text { and } \quad-\circ \Psi: \mathcal{A} \text {-mod } \rightarrow \mathcal{C} \text {-mod. }
$$

These latter functors define inverse equivalences as required.

Conversely, let $\Phi: \mathcal{C}$-mod $\rightarrow \mathcal{A}$-mod and $\Psi: \mathcal{A}$-mod $\rightarrow \mathcal{C}$-mod be mutually inverse equivalences. They restrict to equivalences between $\mathcal{C}$-proj and $\mathcal{A}$-proj.

Let $\dot{i}_{1}, \dot{i}_{2}, \ldots, \dot{i}_{k}$ be a fixed set of representatives of the isomorphism classes of objects in $\mathcal{C}$ and let $\mathcal{C}^{\prime}$ be the full subcategory of $\mathcal{C}$ consisting of these objects. Then $\mathcal{C}^{\prime}$ is a skeleton of $\mathcal{C}$ and hence is equivalent to $\mathcal{C}$. Let $\mathcal{X}_{\mathcal{C}}$ be the full subcategory of $\mathcal{C}$-proj with objects $\mathcal{C}\left(i_{t},{ }_{-}\right)$for $t=1,2, \ldots, k$. The Yoneda isomorphism gives an isomorphism of categories between $\mathcal{X}_{\mathcal{C}}$ and $\left(\mathcal{C}^{\prime}\right)^{\text {op }}$. Similarly we define $\mathcal{A}^{\prime}$ and $\mathcal{X}_{\mathcal{A}}$ and get an isomorphism between $\mathcal{X}_{\mathcal{A}}$ and $\left(\mathcal{A}^{\prime}\right)^{\text {op }}$. Note that $\mathcal{X}_{\mathcal{C}}$ is a multiplicity free additive generator of $\mathcal{C}$-proj. The equivalence $\Phi$ thus maps it to a multiplicity free additive generator of $\mathcal{A}$-proj. Hence $\Phi\left(\mathcal{X}_{\mathcal{C}}\right)$ is isomorphic to $\mathcal{X}_{\mathcal{A}}$ which implies that the categories $\mathcal{C}^{\prime}$ and $\mathcal{A}^{\prime}$ are isomorphic. Therefore $\mathcal{C}$ and $\mathcal{A}$ are equivalent.

In the general case we have the following.

Theorem 2 (Morita Theorem for $\mathbb{k}$-linear categories). Let $\mathcal{A}$ and $\mathcal{C}$ be two finitary $\mathbb{k}$-linear categories. Then the following assertions are equivalent.

(a) There exists a progenerator $\mathcal{P} \in \mathcal{C}$-mod such that $\mathcal{P}^{\text {op }}$ is equivalent to $\mathcal{A}$.

(b) The categories $\mathcal{A}$-proj and $\mathcal{C}$-proj are equivalent. 
(c) The categories $\mathcal{A}$-mod and $\mathcal{C}$-mod are equivalent.

Proof. Claim (c) implies claim (b) as any categorical equivalence sends projective objects to projective objects. If $\Phi: \mathcal{A}$-proj $\rightarrow \mathcal{C}$-proj is an equivalence, then the image of the full subcategory in $\mathcal{A}$-mod with objects $\mathcal{A}\left(i,{ }_{-}\right)$, i $\in \mathcal{A}$, under $\Phi$ is a progenerator for $\mathcal{C}$-proj. Hence claim (b) implies claim (a). Finally, if there exists a progenerator $\mathcal{P} \in \mathcal{C}$-mod such that $\mathcal{P}^{\text {op }}$ is equivalent to $\mathcal{A}$, then the functors

$$
\mathcal{P} \otimes_{\mathcal{A}-}: \mathcal{A} \text {-mod } \rightarrow \mathcal{C} \text {-mod } \quad \text { and } \quad \operatorname{Hom}_{\mathcal{C}}\left(\mathcal{P},_{-}\right): \mathcal{C} \text {-mod } \rightarrow \mathcal{A} \text {-mod }
$$

are easily seen to be mutually inverse equivalences of categories (for the definition of $\mathcal{P} \otimes_{\mathcal{A}}$ - we refer to e.g. [MOS, Page 1139]).

2.4. Image of a functor. Let $\mathcal{A}$ and $\mathcal{C}$ be two categories and $\mathrm{F}: \mathcal{A} \rightarrow \mathcal{C}$ be a functor. In general, one cannot speak about the "image" of F. However, if both $\mathcal{A}$ and $\mathcal{C}$ are small and $\mathrm{F}$ induces an injective map from objects of $\mathcal{A}$ to objects of $\mathcal{C}$, then we can define the image $\mathrm{F}(\mathcal{A})$ of $\mathrm{F}$ to be the subcategory of $\mathcal{C}$ which consists of all objects $\mathrm{F}(\mathrm{i})$ for $i \in \mathcal{A}$ and all morphisms $\mathrm{F}(\alpha)$ where $\alpha$ is a morphism in $\mathcal{A}$.

\section{Finitary 2-CATEgories AND THEIR 2-REPRESENTATIONS}

3.1. Various 2-categories. In this paper by a 2-category we mean a strict locally small 2-category (see [Le] for a concise introduction to 2-categories and bicategories). Let $\mathscr{C}$ be a 2 -category. We will use $i, j, \ldots$ to denote objects in $\mathscr{C}$; 1-morphisms in $\mathscr{C}$ will be denoted by F, G, ..; 2-morphisms in $\mathscr{C}$ will be denoted by $\alpha, \beta, \ldots$ For $i \in \mathscr{C}$ we denote by $\mathbb{1}_{i}$ the corresponding identity 1 -morphism. For a 1-morphism $\mathrm{F}$ we denote by $\mathrm{id}_{\mathrm{F}}$ the corresponding identity 2-morphism. We will write $\circ_{0}$ for horizontal composition of 2-morphisms and $\circ_{1}$ for vertical composition of 2-morphisms. The opposite 2 -category $\mathscr{C}^{\mathrm{op}}$ is obtained by reversing all 1-morphisms and keeping the direction of all 2-morphisms.

Denote by Cat the 2-category of all small categories. Let $\mathbb{k}$ be an algebraically closed field. Denote by $\mathfrak{A}_{\mathbb{k}}$ the 2 -category whose objects are small $\mathbb{k}$-linear fully additive categories, by which we mean additive categories which are idempotentclosed (or Karoubian); 1-morphisms are additive $\mathbb{k}$-linear functors and 2-morphisms are natural transformations. Denote by $\mathfrak{A}_{\mathrm{k}}^{f}$ the full 2-subcategory of $\mathfrak{A}_{\mathbb{k}}$ whose objects are fully additive categories $\mathcal{A}$ such that $\mathcal{A}$ has only finitely many isomorphism classes of indecomposable objects and all morphism spaces in $\mathcal{A}$ are finite dimensional. We also denote by $\mathfrak{R}_{\mathrm{k}}$ the full subcategory of $\mathfrak{A}_{\mathbb{k}}$ containing all objects which are equivalent to $A$-mod for some finite dimensional associative $\mathbb{k}$-algebra $A$.

3.2. Finitary 2-categories. A 2-category $\mathscr{C}$ is called finitary (over $\mathbb{k}$ ), see [MM1], if the following conditions are satisfied:

- $\mathscr{C}$ has finitely many objects up to equivalence;

- for any $i, j \in \mathscr{C}$ we have $\mathscr{C}(i, j) \in \mathfrak{A}_{\mathrm{k}}^{f}$ and horizontal composition is both additive and $\mathbb{k}$-linear;

- for any $i \in \mathscr{C}$ the 1 -morphism $\mathbb{1}_{i}$ is indecomposable. 
3.3. Homomorphisms, strong transformations and modifications. Here we closely follow [Le]. Let $\mathscr{A}$ and $\mathscr{C}$ be 2-categories. A homomorphism $\mathbf{H}: \mathscr{A} \rightarrow \mathscr{C}$ consists of the following data:

- a map $\mathbf{H}$ from objects of $\mathscr{A}$ to objects of $\mathscr{C}$;

- functors $\mathbf{H}_{\mathrm{i}, \mathrm{j}}: \mathscr{A}(\mathrm{i}, \mathrm{j}) \rightarrow \mathscr{C}(\mathbf{H}(\mathrm{i}), \mathbf{H}(\mathrm{j}))$;

- natural isomorphisms

$\mathbf{h}_{\mathrm{G}, \mathrm{F}}: \mathbf{H}_{\mathrm{i}, j}(\mathrm{G}) \circ \mathbf{H}_{\mathrm{i}, \mathrm{j}}(\mathrm{F}) \rightarrow \mathbf{H}_{\mathrm{i}, \mathrm{j}}(\mathrm{G} \circ \mathrm{F}) \quad$ and $\quad \mathbf{h}_{\mathrm{i}}: \mathbb{1}_{\mathbf{H}(\mathrm{i})} \rightarrow \mathbf{H}_{\mathrm{i}, \mathrm{i}}\left(\mathbb{1}_{\mathrm{i}}\right) ;$

such that the following conditions are satisfied:

$$
\begin{aligned}
\mathbf{h}_{\mathrm{H} \circ \mathrm{G}, \mathrm{F}} \circ_{1}\left(\mathbf{h}_{\mathrm{H}, \mathrm{G}} \circ_{0} \operatorname{id}_{\mathbf{H}_{\mathrm{i}, \mathrm{j}}(\mathrm{F})}\right) & =\mathbf{h}_{\mathrm{H}, \mathrm{G} \circ \mathrm{F}} \circ_{1}\left(\mathrm{id}_{\mathbf{H}_{\mathrm{k}, \mathrm{l}}(\mathrm{H})} \circ_{0} \mathbf{h}_{\mathrm{G}, \mathrm{F}}\right), \\
\operatorname{id}_{\mathbf{H}_{\mathrm{i}, j}(\mathrm{~F})} & =\mathbf{h}_{\mathrm{F}, \mathbb{1}_{1}} \circ_{1}\left(\mathrm{id}_{\mathbf{H}_{\mathrm{i}, j}(\mathrm{~F})} \circ_{0} \mathbf{h}_{\mathrm{i}}\right), \\
\operatorname{id}_{\mathbf{H}_{\mathrm{i}, j}(\mathrm{~F})} & =\mathbf{h}_{\mathbb{1}_{j}, \mathrm{~F}} \circ_{1}\left(\mathbf{h}_{\mathrm{j}} \circ_{0} \mathrm{id}_{\mathbf{H}_{\mathrm{i}, j}(\mathrm{~F})}\right) .
\end{aligned}
$$

Given homomorphisms $\mathbf{H}$ and $\mathbf{G}$ from $\mathscr{A}$ to $\mathscr{C}$ a strong transformation $\Phi: \mathbf{H} \rightarrow \mathbf{G}$ is given by the following data:

- functors $\Phi_{\mathrm{i}}: \mathbf{H}(\mathrm{i}) \rightarrow \mathbf{G}(\mathrm{i})$;

- natural isomorphisms $\varphi_{\mathrm{F}}: \mathbf{G}(\mathrm{F}) \circ \Phi_{\mathrm{i}} \rightarrow \Phi_{\mathrm{j}} \circ \mathbf{H}(\mathrm{F})$;

such that the following conditions are satisfied:

$$
\begin{aligned}
\varphi_{\mathrm{G} \circ \mathrm{F}} \circ_{1}\left(\mathrm{~g}_{\mathrm{G}, \mathrm{F}} \circ_{0} \mathrm{id}_{\Phi_{\mathrm{i}}}\right) & =\left(\operatorname{id}_{\Phi_{\mathrm{k}}} \circ_{0} \mathbf{h}_{\mathrm{G}, \mathrm{F}}\right) \circ_{1}\left(\varphi_{\mathrm{G}} \circ_{0} \operatorname{id}_{\mathbf{H}(\mathrm{F})}\right) \circ_{1}\left(\operatorname{id}_{\mathrm{G}(\mathrm{G})} \circ_{0} \varphi_{\mathrm{F}}\right) ; \\
\operatorname{id}_{\Phi_{\mathrm{i}}} \circ_{0} \mathbf{h}_{\mathrm{i}} & =\varphi_{\mathbb{1}_{\mathrm{i}}} \circ_{1}\left(\mathrm{~g}_{\mathrm{i}} \circ_{0} \operatorname{id}_{\Phi_{\mathrm{i}}}\right) .
\end{aligned}
$$

If the natural isomorphisms $\varphi_{\mathrm{F}}$ are identities, the strong transformation is called a strict transformation.

Given two strong transformations $\Phi, \Psi: \mathbf{H} \rightarrow \mathbf{G}$ a modification $\theta: \Phi \rightarrow \Psi$ is a collection of 2-morphisms $\theta_{\mathrm{i}}: \Phi_{\mathrm{i}} \rightarrow \Psi_{\mathrm{i}}$ such that

$$
\psi_{\mathrm{F}} \circ_{1}\left(\operatorname{id}_{\mathbf{G}(\mathrm{F})} \circ_{0} \theta_{\dot{i}}\right)=\left(\theta_{\mathbf{j}} \circ_{0} \operatorname{id}_{\mathbf{H}(\mathrm{F})}\right) \circ_{1} \varphi_{\mathrm{F}} .
$$

3.4. 2-representations. Let $\mathscr{C}$ be a finitary 2-category. We define the following 2-categories of 2-representations of $\mathscr{C}$ :

- the 2-category $\mathscr{C}$-MOD has as objects all homomorphisms from $\mathscr{C}$ to Cat, as 1-morphisms all strong transformations and as 2-morphisms all modifications;

- the 2-category $\mathscr{C}$-amod has as objects all homomorphisms from $\mathscr{C}$ to $\mathfrak{A}_{\mathrm{k}}$, as 1-morphisms all strong transformations and as 2-morphisms all modifications;

- the 2-category $\mathscr{C}$-afmod has as objects all homomorphisms from $\mathscr{C}$ to $\mathfrak{A}_{\mathrm{lk}}^{f}$, as 1-morphisms all strong transformations and as 2-morphisms all modifications;

- the 2-category $\mathscr{C}$-mod has as objects all homomorphisms from $\mathscr{C}$ to $\mathfrak{R}_{\mathbb{k}}$, as 1morphisms all strong transformations and as 2-morphisms all modifications. 
These are indeed 2-categories since $\mathfrak{A}_{\mathbb{k}}, \mathfrak{A}_{\mathbb{k}}^{f}$ and $\mathfrak{R}_{\mathbb{k}}$ are. We will write Hom $\mathscr{C}_{\mathscr{C}}$ for $\operatorname{Hom}_{\mathscr{C} \text {-afmod. }}$

3.5. Biequivalence. Let $\mathscr{A}$ and $\mathscr{C}$ be two 2-categories. A biequivalence $\mathbf{H}: \mathscr{A} \rightarrow \mathscr{C}$ is a homomorphism which is essentially surjective on objects and which is a local equivalence, that is $\mathbf{H}_{i, j}$ is an equivalence for all $i$ and $j$. The 2-categories $\mathscr{A}$ and $\mathscr{C}$ are called biequivalent if there is a biequivalence from $\mathscr{A}$ to $\mathscr{C}$. Biequivalence is an equivalence relation, see [Le, Subsection 2.2].

Alternatively, two homomorphisms $\mathbf{H}: \mathscr{A} \rightarrow \mathscr{C}$ and $\mathbf{G}: \mathscr{C} \rightarrow \mathscr{A}$ are mutually inverse biequivalences if there exist strong transformations

(1) $\mathbf{H} \circ \mathbf{G} \stackrel{\Phi_{1}}{\longrightarrow} \operatorname{Id}_{\mathscr{C}}, \quad \operatorname{Id}_{\mathscr{C}} \stackrel{\Phi_{2}}{\longrightarrow} \mathbf{H} \circ \mathbf{G}, \quad \mathbf{G} \circ \mathbf{H} \stackrel{\Psi_{1}}{\longrightarrow} \operatorname{Id}_{\mathscr{A}} \quad$ and $\quad \operatorname{Id}_{\mathscr{A}} \stackrel{\Psi_{2}}{\longrightarrow} \mathbf{G} \circ \mathbf{H}$ and modifications

$$
\begin{array}{ll}
\theta_{1}: \Phi_{1} \circ \Phi_{2} \rightarrow \mathbb{1}_{\operatorname{Id}_{\mathscr{C}},} & \theta_{2}: \mathbb{1}_{\mathbf{H} \circ \mathbf{G}} \rightarrow \Phi_{2} \circ \Phi_{1}, \\
\theta_{3}: \Psi_{1} \circ \Psi_{2} \rightarrow \mathbb{1}_{\operatorname{Id}_{\mathscr{A}}}, & \theta_{4}: \mathbb{1}_{\mathbf{G} \circ \mathbf{H}} \rightarrow \Psi_{2} \circ \Psi_{1} .
\end{array}
$$

such that the latter are isomorphisms.

3.6. Cancellative envelope of a 2-category. Let $\mathscr{C}$ be a finitary 2-category. Define a new 2-category $\widehat{\mathscr{C}}$ as follows:

- $\widehat{\mathscr{C}}$ has the same objects as $\mathscr{C}$;

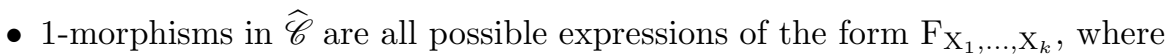
$k \in \mathbb{N}$ and $\mathrm{F}, \mathrm{X}_{1}, \ldots, \mathrm{X}_{k}$ are 1-morphisms in $\mathscr{C}$ such that $\mathrm{F}=\mathrm{X}_{1} \circ \cdots \circ \mathrm{X}_{k}$, and also all possible expressions of the form $\left(\mathbb{1}_{i}\right)_{\varnothing}$, where $i \in \mathscr{C}$;

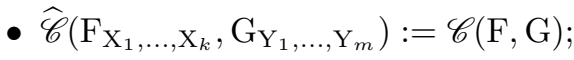

- the identity 1-morphisms are $\left(\mathbb{1}_{i}\right)_{\varnothing}$, where $i \in \mathscr{C}$;

- horizontal composition of 1-morphisms is defined via

$$
\mathrm{F}_{\mathrm{X}_{1}, \ldots, \mathrm{X}_{k}} \circ \mathrm{G}_{\mathrm{Y}_{1}, \ldots, \mathrm{Y}_{m}}:=(\mathrm{F} \circ \mathrm{G})_{\mathrm{X}_{1}, \ldots, \mathrm{X}_{k}, \mathrm{Y}_{1}, \ldots, \mathrm{Y}_{m}}
$$

- both horizontal and vertical composition of 2-morphisms are induced from the corresponding compositions in $\mathscr{C}$.

Forgetting the subscripts of 1-morphisms defines a 2 -functor from $\widehat{\mathscr{C}}$ to $\mathscr{C}$ which is a biequivalence by construction. The category $\widehat{\mathscr{C}}$ is cancellative in the sense that for any 1-morphisms $\mathrm{F}, \mathrm{G}$ and $\mathrm{H}$ in $\widehat{\mathscr{C}}$ the equality $\mathrm{F} \circ \mathrm{H}=\mathrm{G} \circ \mathrm{H}$ implies $\mathrm{F}=\mathrm{G}$ and, moreover, the equality $\mathrm{H} \circ \mathrm{F}=\mathrm{H} \circ \mathrm{G}$ implies $\mathrm{F}=\mathrm{G}$.

3.7. 2-representations of biequivalent 2-categories. We will need the following observation.

Proposition 3. Let $\mathscr{A}$ and $\mathscr{C}$ be two biequivalent finitary 2-categories. Then the 2 -categories $\mathscr{A}$-afmod and $\mathscr{C}$-afmod are biequivalent.

Proof. Every homomorphism $\mathbf{H}: \mathscr{A} \rightarrow \mathscr{C}$ induces a homomorphism

$$
\text { - } \circ \mathbf{H}: \mathscr{C} \text {-afmod } \rightarrow \mathscr{A} \text {-afmod. }
$$


If $\mathbf{H}: \mathscr{A} \rightarrow \mathscr{C}$ and $\mathbf{G}: \mathscr{C} \rightarrow \mathscr{A}$ are inverse biequivalences, then we claim that $\_\circ \mathbf{H}$ and _ $\circ \mathbf{G}$ are also inverse biequivalences. Given the data of (1) and (2), composition with $\operatorname{Id}_{\mathscr{C}}$-afmod and $\operatorname{Id}_{\mathscr{A} \text {-afmod }}$ defines the data establishing biequivalences between $\mathscr{A}$-afmod and $\mathscr{C}$-afmod.

Combining Proposition 3 with construction of the cancellative envelope in Subsection 3.7, we may, without loss of generality, always assume that $\mathscr{C}$ is cancellative.

\section{Projective 2-Representations}

4.1. Principal 2-representations. Let $\mathscr{C}$ be a finitary 2-category. For any i $\in \mathscr{C}$ we have the principal (finitary) 2-representation $\mathscr{C}\left(i,{ }_{-}\right)$of $\mathscr{C}$ which we denote by $\mathbb{P}_{i}$. For any $\mathbf{M} \in \mathscr{C}$-afmod we have the Yoneda equivalence of categories

$$
\operatorname{Hom}_{\mathscr{C} \text {-afmod }}\left(\mathbb{P}_{\mathbf{i}}, \mathbf{M}\right) \cong \mathbf{M}(\mathbf{i})
$$

given by evaluation at $\mathbb{1}_{i}$, which is, moreover, surjective on objects (the proof is analogous to [MM3, Lemma 3]). The direct sum of principal 2-representations with every $i \in \mathscr{C}$ occurring exactly once is the 2 -analog of a "free module of rank one" in classical representation theory.

For $\mathbf{M} \in \mathscr{C}$-afmod consider the 2-category $\mathscr{M}$ defined as follows:

- objects of $\mathscr{M}$ are $\left(\eta, \Phi, \Phi^{\prime}, \mathbb{P}\right)$ where $\mathbb{P}$ is a direct sum of principal 2representations, $\Phi, \Phi^{\prime}: \mathbb{P} \rightarrow \mathbf{M}$ are strong transformations and $\eta: \Phi \rightarrow \Phi^{\prime}$ is a modification;

- a 1-morphism from $\left(\eta, \Phi, \Phi^{\prime}, \mathbb{P}\right)$ to $\left(\zeta, \Psi, \Psi^{\prime}, \mathbb{P}^{\prime}\right)$ is a strong transformation $\Lambda: \mathbb{P} \rightarrow \mathbb{P}^{\prime}$ such that $\Psi \circ \Lambda=\Phi, \Psi^{\prime} \circ \Lambda=\Phi^{\prime}$ and $\zeta \circ_{0} \operatorname{id}_{\Lambda}=\eta$;

- a 2-morphism of $\mathscr{M}$ is just a modification $\lambda: \Lambda \rightarrow \Lambda^{\prime}$ such that $\zeta \circ_{0} \lambda=\eta$.

The 2-category $\mathscr{M}$ is the 2-category of principal covers of $\mathbf{M}$, from which, as we will see below in Proposition 4, we can recover $\mathbf{M}$ as a colimit. This will be important in obtaining a description of projective 2-representations in Proposition 5 and is to be thought of as a substitute for "having enough projective modules" in classical representation theory.

Define a 2 -functor $\Upsilon$ from $\mathscr{M}$ to $\mathscr{C}$-afmod by sending $\left(\eta, \Phi, \Phi^{\prime}, \mathbb{P}\right)$ to $\mathbb{P}$ and defining $\Upsilon$ as the identity on both 1-morphisms and 2-morphisms. Recall that a (strict) cocone $(\mathbf{N}, \mathfrak{W})$ of $\Upsilon$ is an object $\mathbf{N} \in \mathscr{C}$-afmod together with, for any $\left(\eta, \Phi, \Phi^{\prime}, \mathbb{P}\right) \in \mathscr{M}$, an assignment of two strong transformations $\Theta_{1}, \Theta_{2}: \Upsilon\left(\eta, \Phi, \Phi^{\prime}, \mathbb{P}\right) \rightarrow \mathbf{N}$ and a modification $\theta: \Theta_{1} \rightarrow \Theta_{2}$ i.e.

$$
\mathfrak{W}_{\left(\eta, \Phi, \Phi^{\prime}, \mathbb{P}\right)}=\quad \mathbb{P} \frac{\Theta_{1}}{\frac{\Downarrow \theta_{\eta}}{\Theta_{2}}} \mathbf{N}
$$

such that for all 2-morphisms $\lambda: \Lambda \rightarrow \Lambda^{\prime}$ in $\mathscr{M}$ as above, we have $\theta_{\zeta} \circ_{0} \lambda=\theta_{\eta}$. A strict colimit of $\Upsilon$ is an initial object in the 2-category of cocones of $\Upsilon$. Clearly, the pair $(\mathbf{M}, \mathfrak{V})$ defined via $\mathfrak{V}_{\left(\eta, \Phi, \Phi^{\prime}, \mathbb{P}\right)}:=\left(\Phi \stackrel{\eta}{\Longrightarrow} \Phi^{\prime}\right)$ is a strict cocone of $\Upsilon$.

Proposition 4. The cocone $(\mathbf{M}, \mathfrak{V})$ is a strict colimit. 
Proof. Let $(\mathbf{N}, \mathfrak{W})$ be another strict cocone of $\Upsilon$ in $\mathscr{C}$-afmod. To define a strong transformation $\Theta: \mathbf{M} \rightarrow \mathbf{N}$, let $\mathbf{i} \in \mathscr{C}, X, Y \in \mathbf{M}(\mathbf{i})$ and $f: X \rightarrow Y$. Consider $\left(\eta, \Phi, \Phi^{\prime}, \mathbb{P}_{\mathrm{i}}\right)$ such that $\Phi$ is a strict transformation sending $\mathbb{1}_{\mathrm{i}}$ to $X, \Phi^{\prime}$ is a strict transformation sending $\mathbb{1}_{i}$ to $Y$ and $\eta$ is a modification which evaluates to $f$ (these exist by the Yoneda lemma). Due to strictness of $(\mathbf{N}, \mathfrak{W})$ as a cocone, we necessarily have $\Theta(X \stackrel{f}{\longrightarrow} Y)=\mathfrak{W}_{\left(\eta, \Phi, \Phi^{\prime}, \mathbb{P}_{\mathbf{i}}\right)}\left(\mathbb{1}_{\mathbf{i}}\right)$. On the other hand, the latter defines a morphism from $(\mathbf{M}, \mathfrak{V})$ to $(\mathbf{N}, \mathfrak{W})$. The claim follows.

4.2. Projective 2-representations. Let $\mathscr{C}$ be a finitary 2-category. A finitary 2-representation $\mathbf{P}$ of $\mathscr{C}$ is called projective if $\operatorname{Hom}_{\mathscr{C}}\left(\mathbf{P},,_{-}\right)$preserves all small colimits.

Proposition 5. A finitary 2-representation $\mathbf{P}$ of $\mathscr{C}$ is projective if and only if it is a retract of a direct sum of principal 2-representations.

Proof. We follow the classical argument from [BD, Proposition 2]. From the Yoneda lemma it follows that all principal representations (and their direct sums) are projective. Let $\mathbb{P}$ be a direct sum of principal 2-representations and $\mathbf{P}$ a retract of $\mathbb{P}$, that is there exist $\Phi: \mathbb{P} \rightarrow \mathbf{P}$ and $\Psi: \mathbf{P} \rightarrow \mathbb{P}$ such that $\Phi \Psi$ is isomorphic to the identity on $\mathbf{P}$. Then $\operatorname{Hom}_{\mathscr{C}}\left(\mathbf{P},_{-}\right)$is isomorphic to $\operatorname{Hom}_{\mathscr{C}}\left(\mathbb{P},_{-}\right) \circ \Psi$ and hence commutes with all small colimits $\left(\operatorname{as~}_{\operatorname{Hom}}\left(\mathbb{P},_{-}\right)\right.$does).

Let now $\mathbf{M}$ be a projective finitary 2-representation of $\mathscr{C}$. By Proposition $4, \mathbf{M}$ is equivalent to the colimit of $\Upsilon: \mathscr{M} \rightarrow \mathscr{C}$-afmod. Since $\operatorname{Hom}_{\mathscr{C}}\left(\mathbf{M},{ }_{-}\right)$preserves all small colimits, we have

$$
\operatorname{Hom}_{\mathscr{C}}(\mathbf{M}, \mathbf{M}) \cong \operatorname{Hom}_{\mathscr{C}}\left(\mathbf{M}, \lim _{\rightarrow} \Upsilon\right) \cong \lim _{\rightarrow} \operatorname{Hom}_{\mathscr{C}}(\mathbf{M}, \Upsilon)
$$

Via this equivalence, the identity on $\mathbf{M}$ thus must have a representative in the right hand side. This means that there exists a direct sum $\mathbb{P}$ of principal 2-representations and $\Phi: \mathbb{P} \rightarrow \mathbf{M}$ such that the identity on $\mathbf{M}$ is represented in the term indexed by $\left(\operatorname{id}_{\Phi}, \Phi, \Phi, \mathbb{P}\right)$, say by some $\Psi$. This means that $\Phi \Psi$ is the identity on $\mathbf{M}$. The claim follows.

4.3. Idempotent matrices with non-negative integer coefficients. For an arbitrary $n \in \mathbb{N}$ we denote by $0_{n}$ the zero $n \times n$ matrix and by $1_{n}$ the identity $n \times n$ matrix. We will need the following result from [Fl, Theorem 2]:

Proposition 6. Let $M$ be an idempotent matrix with non-negative integer coefficients. Then there is a permutation matrix $S$ such that $S^{-1} M S$ has the form

$$
\left(\begin{array}{ccc}
0_{a} & A & A B \\
0 & 1_{b} & B \\
0 & 0 & 0_{c}
\end{array}\right)
$$

for some matrices $A$ and $B$.

4.4. Idempotent endomorphisms of principal representations. By (3), for every $i, j \in \mathscr{C}$ we have $\operatorname{Hom}_{\mathscr{C} \text {-afmod }}\left(\mathbb{P}_{i}, \mathbb{P}_{j}\right) \cong \mathscr{C}(j, i)$, which means that every homomorphism from $\mathbb{P}_{i}$ to $\mathbb{P}_{j}$ is isomorphic to right multiplication by some 1-morphism in $\mathscr{C}(\mathrm{j}, \mathrm{i})$. Similarly, given a finite direct sum of principal 2representations (with, say, $k$ summands), every endomorphism $\Phi$ of this direct sum is isomorphic to right multiplication by a $k \times k$ matrix whose coefficients are appropriate 1-morphisms in $\mathscr{C}$. We will call a summand of any entry in this matrix a summand of $\Phi$. 
Let $\mathbf{P}=\bigoplus_{s=1}^{k} \mathbb{P}_{\mathbf{i}_{s}}$ be a finite direct sum of principal 2-representations of $\mathscr{C}$ and $\Phi \in \operatorname{End}_{\mathscr{C} \text {-afmod }}(\mathbf{P})$. We associate with $\Phi$ a matrix $M:=M_{\Phi}$ defined as follows: the matrix $M$ is a block $k \times k$ matrix with blocks indexed by the summands of P. The rows of the $(r, s)$-block (i.e. block-row index $r$ and block-column index $s$ ) are indexed by indecomposable 1 -morphisms in $\mathscr{C}\left(\mathbf{i}_{r}, \mathbf{j}\right)$ for any $\mathrm{j}$ in $\mathscr{C}$. The columns of the $(r, s)$-block are indexed by indecomposable 1-morphisms in $\mathscr{C}\left(i_{s}, j\right)$ for any $\mathrm{j}$ in $\mathscr{C}$. Let $\mathrm{F}$ be an indecomposable 1-morphism in $\mathscr{C}\left(\mathbf{i}_{r}, j\right)$ and $\mathrm{G}$ be an indecomposable 1-morphism in $\mathscr{C}\left(\mathbf{i}_{s}, \mathbf{j}^{\prime}\right)$. Then the $(\mathrm{F}, \mathrm{G})$-entry in the $(r, s)$-block is given by the multiplicity of $\mathrm{G}$ as a direct summand of $\Phi(\mathrm{F}$ ) (in particular, if this entry is nonzero, then $\left.\mathrm{j}=\mathrm{j}^{\prime}\right)$. Note that $M$ is a square matrix with non-negative integer coefficients. Furthermore, if $\Psi \in \operatorname{End}_{\mathscr{C} \text {-afmod }}(\mathbf{P})$, then $M_{\Phi} M_{\Psi}=M_{\Psi \circ \Phi}$. In particular, $\Psi$ is idempotent, by which we mean $\Psi^{2} \cong \Psi$, if and only if we have $M_{\Psi}^{2}=M_{\Psi}$.

Assume that $\Phi \cong \Phi^{2} \neq 0$, then $M \neq 0$. By Proposition 6 , in this case all diagonal entries of $M$ are equal to either 0 or 1 and there is at least one non-zero diagonal entry. Let $\mathrm{F}_{1}, \mathrm{~F}_{2}, \ldots, \mathrm{F}_{m}$ be a complete list of indecomposable 1-morphisms indexing the non-zero diagonal entries of $M$.

Lemma 7. (a) For every $i \in\{1,2, \ldots, m\}$, let $\mathrm{j}_{i}, \mathrm{k}_{i}$ be such that $\mathrm{F}_{i} \in \mathscr{C}\left(\mathrm{j}_{i}, \mathrm{k}_{i}\right)$. Then there is a unique indecomposable summand $\Gamma_{i}$ of $\Phi$, given by right multiplication by an indecomposable 1-morphism $\mathrm{G}_{i} \in \mathscr{C}\left(\mathrm{j}_{i}, \mathrm{j}_{i}\right)$, and such that $\Gamma_{i}\left(\mathrm{~F}_{i}\right) \cong \mathrm{F}_{i} \circ \mathrm{G}_{i} \cong \mathrm{F}_{i} \oplus \mathrm{X}_{i}$ for some 1-morphism $\mathrm{X}_{i} \in \mathscr{C}\left(\mathrm{j}_{i}, \mathrm{k}_{i}\right)$.

(b) We have $\Phi(\mathrm{X}) \cong \mathrm{X} \circ \mathrm{G}_{i}=0$.

Proof. We use Proposition 6 to reduce $M$ to the form $\tilde{M}$ given by (4). Then the multiplicities of $\Phi\left(\mathrm{F}_{i}\right)$ are given by the row $v$ of $\tilde{M}$ indexed by $\mathrm{F}_{i}$. Note that $\mathrm{F}_{i}$ indexes a row in the second row of blocks of the $3 \times 3$ block decomposition of $\tilde{M}$. Therefore $\Phi\left(\mathrm{F}_{i}\right)=\mathrm{F}_{i} \oplus \mathrm{Y}$ for some $\mathrm{Y}$ with $\Phi(\mathrm{Y})=0$. Now there is a unique indecomposable summand $\Gamma_{i}$ of $\Phi$ which contributes the summand $\mathrm{F}_{i}$ above, and it is given by right multiplication with a unique indecomposable 1-morphism $\mathrm{G}_{i} \in \mathscr{C}\left(\mathrm{j}_{i}, \mathrm{j}_{i}\right)$. Define $\mathrm{X}_{i}$ via $\mathrm{F}_{i} \circ \mathrm{G}_{i} \cong \mathrm{F}_{i} \oplus \mathrm{X}_{i}$. Then, with $\mathrm{X}_{i}$ being a summand of $\mathrm{Y}$ and $\Gamma_{i}$ being a summand of $\Phi$, we have that $\Gamma_{i}\left(\mathrm{X}_{i}\right)$ is a summand of $\Phi(Y)$ and hence equals zero.

Lemma 8. For $i \in\{1,2, \ldots, m\}$ the 1-morphism $\mathrm{G}_{i}$ satisfies $\mathrm{G}_{i} \circ \mathrm{G}_{i} \cong \mathrm{G}_{i} \oplus \mathrm{Q}_{i}$ such that $\mathrm{G}_{i} \circ \mathrm{Q}_{i}=0$ and $\Phi\left(\mathrm{Q}_{i}\right)=0$.

Proof. From Lemma $7(\mathrm{a})$ we have that $\mathrm{G}_{i}$ is the only indecomposable summand of $\Phi$ sending $\mathrm{F}_{i}$ to $\mathrm{F}_{i}$ (plus something). This yields that $\mathrm{G}_{i}$ is a summand of $\mathrm{G}_{i} \circ \mathrm{G}_{i}$ occurring with multiplicity one (since $\mathrm{F}_{i}$ appears with multiplicity one in $\mathrm{F}_{i} \circ \mathrm{G}_{i}$ ). In particular, it follows that $\mathrm{G}_{i} \cong \mathrm{F}_{j_{i}}$ for some $j_{i} \in\{1,2, \ldots, m\}$. We also have $\mathrm{G}_{j_{i}}=\mathrm{G}_{i}$, so that $\mathrm{Q}_{i}=\mathrm{X}_{j_{i}}$. From Lemma $7(\mathrm{~b})$ we thus get $\Phi\left(\mathrm{Q}_{i}\right)=0$, in particular $\mathrm{Q}_{i} \circ \mathrm{G}_{i}=0$. To prove $\mathrm{G}_{i} \circ \mathrm{Q}_{i}=0$ we compute $\mathrm{G}_{i}^{3}$ in two different ways. On the one hand,

$$
\mathrm{G}_{i}^{3} \cong \mathrm{G}_{i} \circ\left(\mathrm{G}_{i} \oplus \mathrm{Q}_{i}\right) \cong \mathrm{G}_{i} \oplus \mathrm{Q}_{i} \oplus \mathrm{G}_{i} \circ \mathrm{Q}_{i} .
$$

On the other hand,

$$
\mathrm{G}_{i}^{3} \cong\left(\mathrm{G}_{i} \oplus \mathrm{Q}_{i}\right) \circ \mathrm{G}_{i} \cong \mathrm{G}_{i} \oplus \mathrm{Q}_{i} \oplus \mathrm{Q}_{i} \circ \mathrm{G}_{i}
$$

Now $\mathrm{G}_{i} \circ \mathrm{Q}_{i}=0$ follows from $\mathrm{Q}_{i} \circ \mathrm{G}_{i}=0$ by comparing the two isomorphisms above. 
Lemma 9. Let $i, j \in\{1,2, \ldots, m\}$ be such that $\mathrm{G}_{i} \not \mathrm{G}_{j}$.

(a) We have $\Phi\left(\mathrm{G}_{i} \circ \mathrm{G}_{j}\right)=0$.

(b) We have $\mathrm{G}_{i} \circ \mathrm{G}_{j}=0$.

Proof. We use Proposition 6 to reduce $M$ to the form $\tilde{M}$ given by (4). Note that $\mathrm{G}_{i}$ indexes a row in the second block of the $3 \times 3$ block decomposition of $\tilde{M}$, so $\Phi\left(\mathrm{G}_{i}\right)=\mathrm{G}_{i} \oplus \mathrm{Y}$ with $\Phi(\mathrm{Y})=0$. The composition $\mathrm{G}_{i} \circ \mathrm{G}_{j}$ is a direct summand of $\Phi\left(\mathrm{G}_{i}\right)$. Since $\mathrm{G}_{i} \neq \mathrm{G}_{j}$, the composition $\mathrm{G}_{i} \circ \mathrm{G}_{j}$ does not contain $\mathrm{G}_{i}$ as a direct summand (by Lemma 7(a)) and is hence contained in $\mathrm{Y}$, implying that $\Phi\left(\mathrm{G}_{i} \circ \mathrm{G}_{j}\right)=0$. This proves claim (a).

As $\left(\mathrm{G}_{i} \circ \mathrm{G}_{j}\right) \circ \mathrm{G}_{j}$ is a summand of $\Phi\left(\mathrm{G}_{i} \circ \mathrm{G}_{j}\right)$, we obtain $\left(\mathrm{G}_{i} \circ \mathrm{G}_{j}\right) \circ \mathrm{G}_{j}=0$ by (a). On the other hand, $\mathrm{G}_{i} \circ\left(\mathrm{G}_{j} \circ \mathrm{G}_{j}\right)$ contains, as a summand, $\mathrm{G}_{i} \circ \mathrm{G}_{j}$ by Lemma 8 . This implies claim (b) and completes the proof.

Note that it is possible that $\mathrm{G}_{i} \cong \mathrm{G}_{j}$ for $i \neq j$, where $i, j \in\{1,2, \ldots, m\}$. Therefore we define $\Gamma$ to be the multiplicity free direct sum of all $\Gamma_{i}$ for $i \in\{1,2, \ldots, m\}$. Similarly, define $\Theta$ such that $\Gamma^{2} \cong \Gamma \oplus \Theta$. From the above discussion it follows that the endomorphism $\Theta$ is given by putting, for each $i$, a copy of $\mathrm{Q}_{i}$ (as given by Lemma 8) in the appropriate places. Define $\Pi$ to be a summand of $\Phi$ such that $\Phi=\Gamma \oplus \Theta \oplus \Pi$. As an immediate corollary from Lemma 8 we have.

Corollary 10. We have $\Phi \Theta=0$.

Lemma 11. We have the following identities:

(a) $Г \Pi \Gamma=\Theta \Pi=\Gamma \Pi^{2}=\Pi^{2} \Gamma=0$.

(b) $\Pi \cong \Gamma \Pi \oplus \Pi \Gamma \oplus \Pi^{2}$.

(c) $\Pi^{3}=0$.

(d) $\Pi^{2} \cong \Pi \Gamma \Pi$.

Proof. Using Corollary 10, we compute

$$
\Gamma \oplus \Theta \oplus \Pi=\Phi \cong \Phi^{2}=\Gamma \oplus \Theta \oplus \Gamma \Pi \oplus \Pi \Gamma \oplus \Theta \Pi \oplus \Pi^{2}
$$

which implies

$$
\Pi \cong \Gamma \Pi \oplus \Pi \Gamma \oplus \Theta \Pi \oplus \Pi^{2} .
$$

Inserting the right hand side of (5) into the first summand on the right we obtain

$$
\Pi \cong \Gamma\left(\Gamma \Pi \oplus \Pi \Gamma \oplus \Theta \Pi \oplus \Pi^{2}\right) \oplus \Pi \Gamma \oplus \Theta \Pi \oplus \Pi^{2} .
$$

Using Corollary 10 and comparing the right hand sides of (5) and (6) gives the identities ГПГ $=\Gamma \Pi \Theta=\Gamma \Pi^{2}=0$. Inserting the right hand side of (5) into the second summand on the right we obtain

$$
\Pi \cong \Gamma \Pi \oplus\left(\Gamma \Pi \oplus \Pi \Gamma \oplus \Theta \Pi \oplus \Pi^{2}\right) \Gamma \oplus \Theta \Pi \oplus \Pi^{2} .
$$

Using Corollary 10 and comparing the right hand sides of (5) and (7) gives the identities $\Theta \Pi=\Pi^{2} \Gamma=0$. This proves claim (a). Claim (b) follows from (5) and claim (a). 
Let $N$ be the matrix associated to $\Pi$ (similarly to how $M$ is associated to $\Phi$ ). Then both $N$ and $M-N$ have non-negative integer coefficients. Let $S$ be a permutation matrix such that $S^{-1} M S=\tilde{M}$ and set $\tilde{N}=S^{-1} N S$. Then both $\tilde{N}$ and $\tilde{M}-\tilde{N}$ have non-negative integer coefficients and from the definition of $\Pi$ it follows that $\tilde{N}$ has the form

$$
\left(\begin{array}{ccc}
0_{a} & A^{\prime} & B^{\prime} \\
0 & 0_{b} & C^{\prime} \\
0 & 0 & 0_{c}
\end{array}\right) .
$$

Clearly, $\tilde{N}^{3}=0$ and thus $\Pi^{3}=0$ proving claim (c).

Inserting (b) into one of the factors in $\Pi^{2}=\Pi^{2}$, we obtain

$$
\Pi^{2} \cong\left(\Gamma \Pi \oplus \Pi \Gamma \oplus \Pi^{2}\right) \Pi \text {. }
$$

Now claim (d) follows from claims (a) and (c). This completes the proof.

Corollary 12. We have the following isomorphisms:

(a) $(\Gamma \oplus \Theta)^{2} \cong \Gamma \oplus \Theta$.

(b) $(\Gamma \oplus \Theta \oplus \Pi \Gamma)^{2} \cong \Gamma \oplus \Theta \oplus \Pi \Gamma$.

(c) $(\Gamma \oplus \Theta \oplus \Pi \Gamma)(\Gamma \oplus \Theta) \cong \Gamma \oplus \Theta \oplus \Pi \Gamma$.

(d) $(\Gamma \oplus \Theta)(\Gamma \oplus \Theta \oplus \Pi \Gamma) \cong \Gamma \oplus \Theta$.

(e) $\Phi(\Gamma \oplus \Theta \oplus \Pi \Gamma) \cong \Gamma \oplus \Theta \oplus \Pi \Gamma$.

(f) $(\Gamma \oplus \Theta \oplus \Pi \Gamma) \Phi \cong \Phi$.

Proof. Claim (a) follows from the definitions and Corollary 10. Claim (b) follows from claim (a), Corollary 10 and Lemma 11(a). Claim (c) follows from Corollary 10. Claims (d) and (e) follow from Corollary 10 and Lemma 11(a). Finally, claim (f) follows from Corollary 10 and Lemma 11(a), (b) and (d).

4.5. Projective 2-subrepresentations of principal 2-representations. For $i \in \mathscr{C}$ fix an indecomposable 1-morphism $\mathrm{G} \in \mathscr{C}(i, i)$ and also a 1-morphism $\mathrm{Q} \in \mathscr{C}(\mathrm{i}, \mathrm{i})$ such that

$$
\mathrm{G} \circ \mathrm{G} \cong \mathrm{G} \oplus \mathrm{Q} \quad \text { and } \quad \mathrm{G} \circ \mathrm{Q}=\mathrm{Q} \circ \mathrm{G}=\mathrm{Q} \circ \mathrm{Q}=0 .
$$

Then $\mathrm{E}:=\mathrm{G} \circ \mathrm{G}$ is a weakly idempotent 1-morphism in $\mathscr{C}(\mathrm{i}, \mathrm{i}$ ) (in the sense that $\mathrm{E} \circ \mathrm{E} \cong \mathrm{E})$. Assume now that $\mathscr{C}$ is cancellative. In this case the functor $(-\circ \mathrm{E})_{j}$ is injective when restricted to objects of $\mathbb{P}_{i}(j)$. Hence we can consider the corresponding image $\mathbb{P}_{i}(j) \circ \mathrm{E}$. The left action of $\mathscr{C}$ leaves $\mathbb{P}_{i}(-) \circ \mathrm{E}$ invariant. We denote by $\mathbf{P}_{i, \mathrm{E}}$ this 2 -subrepresentation of $\mathbb{P}_{\mathrm{i}}$ and by $\Lambda$ the corresponding natural inclusion. Denote by $\Lambda^{\prime}: \mathbb{P}_{i} \rightarrow \mathbf{P}_{i, E}$ the strict transformation given by sending $\mathbb{1}_{i}$ to E.

Proposition 13. The composition $\Lambda^{\prime} \Lambda$ is isomorphic to the identity on $\mathbf{P}_{i, \mathrm{E}}$. In particular, $\mathbf{P}_{\mathrm{i}, \mathrm{E}}$ is projective.

Proof. Let $\mathrm{F}$ and $\mathrm{F}^{\prime}$ be two 1-morphisms in $\mathscr{C}$. Then $\mathrm{F} \circ \mathrm{E}$ and $\mathrm{F}^{\prime} \circ \mathrm{E}$ are in $\mathbf{P}_{\mathrm{i}, \mathrm{E}}$. Any morphism $\alpha: \mathrm{F} \circ \mathrm{E} \rightarrow \mathrm{F}^{\prime} \circ \mathrm{E}$ in $\mathbf{P}_{i, \mathrm{E}}$ is, by definition, of the form $\beta \circ_{0} i_{\mathrm{E}}$ 
for some $\beta: \mathrm{F} \rightarrow \mathrm{F}^{\prime}$. The composition $\Lambda^{\prime} \Lambda$ is given by right multiplication by $\mathrm{E}$. Denote by $\eta: \mathrm{E} \rightarrow \mathrm{E}^{2}$ an isomorphism. Then the diagram

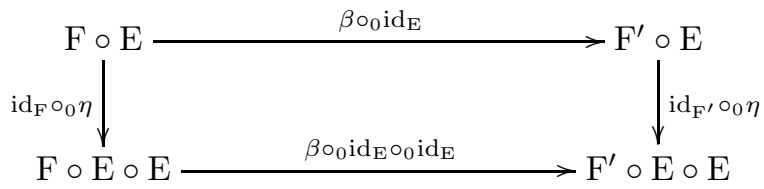

where the vertical arrows are isomorphisms provides an isomorphism from the identity on $\mathbf{P}_{\mathrm{i}, \mathrm{E}}$ to $\Lambda^{\prime} \Lambda$.

Corollary 14. (a) The restriction of _ $\circ \mathrm{E}$ to $\mathbf{P}_{\mathbf{i}, \mathrm{E}}$ is isomorphic to the identity functor on $\mathbf{P}_{\mathrm{i}, \mathrm{E}}$.

(b) The restriction of _ $\circ \mathrm{G}$ to $\mathbf{P}_{\mathrm{i}, \mathrm{E}}$ is isomorphic to the identity functor on $\mathbf{P}_{\mathrm{i}, \mathrm{E}}$.

(c) $\operatorname{End}_{\mathscr{C}}\left(\mathbf{P}_{\mathrm{i}, \mathrm{E}}\right)$ is biequivalent to $\mathrm{E} \circ \mathscr{C}(\mathrm{i}, \mathrm{i}) \circ \mathrm{E}$.

Proof. Claim (a) is a direct consequence of Proposition 13. Claim (b) follows from claim (a) since $\mathrm{E} \circ \mathrm{Q}=0$ (see Lemma 8).

To prove claim (c) we consider the composition

$$
\mathbb{P}_{\mathrm{i}} \stackrel{\Lambda^{\prime}}{\longrightarrow} \mathbf{P}_{\mathrm{i}, \mathrm{E}} \stackrel{\Phi}{\longrightarrow} \mathbf{P}_{\mathrm{i}, \mathrm{E}} \stackrel{\Lambda}{\longrightarrow} \mathbb{P}_{\mathrm{i}}
$$

where $\Phi \in \operatorname{End}_{\mathscr{C}}\left(\mathbf{P}_{i, E}\right)$. Then $\Lambda \Phi \Lambda^{\prime}$ is an endomorphism of $\mathbb{P}_{i}$ and hence is given (up to equivalence) by right multiplication with some 1 -morphism $\mathrm{F} \in \mathscr{C}(\mathbf{i}, \mathbf{i})$ by the Yoneda Lemma. We have $\mathrm{F} \circ \mathrm{E} \cong \mathrm{F}$ since $\mathrm{F} \in \mathbf{P}_{\mathrm{i}, \mathrm{E}}$ by claim (a). Further, using idempotency of $\mathrm{E}$ we have

$$
\mathrm{F} \cong \Phi(\mathrm{E}) \cong \Phi(\mathrm{E} \circ \mathrm{E}) \cong \mathrm{E} \circ \Phi(\mathrm{E}) \cong \mathrm{E} \circ \mathrm{F} .
$$

This yields that $\mathrm{F}$ is isomorphic to a 1-morphism in $\mathrm{E} \circ \mathscr{C}(\mathrm{i}, \mathrm{i}) \circ \mathrm{E}$. If $\Phi^{\prime} \in$ $\operatorname{End}_{\mathscr{C}}\left(\mathbf{P}_{\mathrm{i}, \mathrm{E}}\right)$ is similarly given by some $\mathrm{F}^{\prime}$ and $\eta: \Phi \rightarrow \Phi^{\prime}$ is a modification, then, again by the Yoneda Lemma, the corresponding modification

$$
\operatorname{id}_{\Lambda} \circ_{0} \eta \circ_{0} \operatorname{id}_{\Lambda^{\prime}}: \Lambda \Phi \Lambda^{\prime} \rightarrow \Lambda \Phi^{\prime} \Lambda^{\prime}
$$

is given by some 2-morphism $\alpha: \mathrm{F} \rightarrow \mathrm{F}^{\prime}$. It follows that $\eta$ is given by restriction of $\alpha$ to $\mathbf{P}_{\mathrm{i}, \mathrm{E}}$. Claim (c) follows.

4.6. Description of projective finitary 2-representations. Now we are ready to describe projective 2-representations of $\mathscr{C}$. We assume that $\mathscr{C}$ is cancellative.

Theorem 15. Let $\mathbf{P}$ be a projective 2-representation of $\mathscr{C}$. Then

$$
\mathbf{P} \cong \bigoplus_{s=1}^{m} \mathbf{P}_{\mathrm{i}_{s}, \mathrm{E}_{s}}
$$

for some $\mathrm{i}_{s} \in \mathscr{C}$ and $\mathrm{E}_{s} \in \mathscr{C}\left(\mathrm{i}_{s}, \mathrm{i}_{s}\right)$ such that $\mathrm{E}_{s}=\mathrm{G}_{s} \circ \mathrm{G}_{s}$ for some indecomposable $\mathrm{G}_{s} \in \mathscr{C}\left(\mathrm{i}_{s}, \mathrm{i}_{s}\right)$ and, additionally,

$$
\mathrm{G}_{s} \circ \mathrm{G}_{s} \cong \mathrm{G}_{s} \oplus \mathrm{Q}_{s} \quad \text { and } \quad \mathrm{G}_{s} \circ \mathrm{Q}_{s}=\mathrm{Q}_{s} \circ \mathrm{G}=\mathrm{Q}_{s} \circ \mathrm{Q}_{s}=0
$$

for some $\mathrm{Q}_{s} \in \mathscr{C}\left(\mathrm{i}_{s}, \mathbf{i}_{s}\right)$. 
Proof. Let $\mathbf{P}$ be a projective 2-representation of $\mathscr{C}$. By Proposition 5 , there exists a direct sum $\mathbb{P}$ of principal 2-representations and strong transformations $\Phi: \mathbb{P} \rightarrow \mathbf{P}$ and $\Psi: \mathbf{P} \rightarrow \mathbb{P}$ such that $\Phi \Psi$ is isomorphic to the identity endomorphism of $\mathbf{P}$. Therefore $\Psi \Phi$ is isomorphic to an idempotent endomorphism of $\mathbb{P}$.

By our analysis in Subsection 4.4, $\Psi \Phi$ is of the form $\Psi \Phi \cong \Gamma \oplus \Theta \oplus \Pi$ with $\Gamma^{2} \cong \Gamma \oplus \Theta$ and $\Theta \Psi \Phi=\Psi \Phi \Theta=0$. By Corollary 12(a) and (c), $\Gamma \oplus \Theta$ and $(\Gamma \oplus \Theta \oplus \Pi \Gamma)$ are also idempotent endomorphisms of $\mathbb{P}$. Moreover, by Corollary 12(e) and (f), we have

$$
\begin{gathered}
\Psi \Phi \cong \Psi \Phi(\Gamma \oplus \Theta \oplus \Pi \Gamma) \Psi \Phi \\
\Gamma \oplus \Theta \oplus \Pi \Gamma \cong(\Gamma \oplus \Theta \oplus \Pi \Gamma) \Psi \Phi(\Gamma \oplus \Theta \oplus \Pi \Gamma)
\end{gathered}
$$

and similarly Corollary $12(\mathrm{c})$ and (d) yield

$$
\begin{gathered}
\Gamma \oplus \Theta \oplus \Pi \Gamma \cong(\Gamma \oplus \Theta \oplus \Pi \Gamma)(\Gamma \oplus \Theta)(\Gamma \oplus \Theta \oplus \Pi \Gamma) \\
\Gamma \oplus \Theta \cong(\Gamma \oplus \Theta)(\Gamma \oplus \Theta \oplus \Pi \Gamma)(\Gamma \oplus \Theta) .
\end{gathered}
$$

Consider the strong transformations

$$
\begin{aligned}
& \Omega:=(\Gamma \oplus \Theta)(\Gamma \oplus \Theta \oplus \Pi \Gamma) \Psi: \quad \mathbf{P} \rightarrow \mathbb{P} \\
& \Omega^{\prime}:=\Phi(\Gamma \oplus \Theta \oplus \Pi \Gamma)(\Gamma \oplus \Theta): \mathbb{P} \rightarrow \mathbf{P} .
\end{aligned}
$$

We have $\Omega^{\prime} \Omega \cong \Phi \Psi \Omega^{\prime} \Omega \Phi \Psi$ and the latter is isomorphic to the identity on $\mathbf{P}$ by (10) and (11). In the other direction, we deduce from (10) and (11) that the composition $\Omega \Omega^{\prime}$ is isomorphic to $\Gamma \oplus \Theta$. The latter is a diagonal idempotent endomorphism of $\mathbb{P}$. Since $\mathscr{C}$ is cancellative, we have a well-defined image of $\Gamma \oplus \Theta$ which has the form specified in (9) by Proposition 13. Moreover, $\Gamma \oplus \Theta$ is isomorphic to the identity endomorphism of this image. This completes the proof.

We would like to emphasize here the main difference between classical representation theory and 2-representation theory. In classical representation theory, indecomposable projectives correspond to indecomposable idempotents. In 2-representation theory, the idempotent associated to an indecomposable projective 2-representation might not itself be indecomposable, but is the square of an indecomposable 1morphism (which itself might not be idempotent), see Example 6.2 for an example of this.

\section{2-MORITA THEORY}

5.1. 2-progenerators. Let $\mathscr{C}$ be a finitary 2-category. We denote by $\mathscr{C}$-proj the full 2-subcategory of $\mathscr{C}$-afmod whose objects are projective 2-representations. A full 2-subcategory $\mathscr{P}$ of $\mathscr{C}$-proj is called a 2-progenerator provided that for any projective 2-representation $\mathbf{P}$ of $\mathscr{C}$ there is $\mathbf{P}^{\prime}$ in the additive closure of $\mathscr{P}$ and strong transformations $\Psi: \mathbf{P} \rightarrow \mathbf{P}^{\prime}$ and $\Phi: \mathbf{P}^{\prime} \rightarrow \mathbf{P}$ such that $\Phi \Psi$ is isomorphic to the identity on $\mathbf{P}$. For example, the 2 -subcategory $\mathscr{P}_{\mathscr{C}, \mathbb{P}}$ of $\mathscr{C}$-afmod whose objects are the principal 2-representations of $\mathscr{C}$ is a 2-progenerator. Note that $\mathscr{P}_{\mathscr{C}, \mathbb{P}}$ is biequivalent to $\mathscr{C}^{\mathrm{op}}$ and $\operatorname{End}_{\mathscr{C}}\left(\mathscr{P}_{\mathscr{C}, \mathbb{P}}\right)$ is biequivalent to $\mathscr{C}$. Note also that a full 2 -subcategory $\mathscr{P}$ of $\mathscr{C}$-proj is a progenerator if any principle 2 -representation is a retract of an object in the additive closure of $\mathscr{P}$.

5.2. The essential 2-subcategory of a finitary 2-category. Let $\mathscr{C}$ be a finitary 2-category. Define a binary relation $\preceq$ on the set of equivalence classes of objects of $\mathscr{C}$ as follows: $i \preceq j$ if and only if there exist $\Phi \in \mathscr{C}(j, i)$ and $\Psi \in \mathscr{C}(i, j)$ such that $\Phi \Psi \cong \mathbb{1}_{i}$. Denote by ${ }^{\dagger} \mathscr{C}$ the full 2 -subcategory of $\mathscr{C}$ given by a choice of one 
object $i$ in each equivalence class which is a maximal element with respect to $\preceq$. We will call ${ }^{\dagger} \mathscr{C}$ the essential 2 -subcategory of $\mathscr{C}$.

5.3. The main result. The following is the main result of this paper. Recall that $\mathscr{A}^{\mathrm{op}}$ is defined in Subsection 3.1.

Theorem 16 (Morita theorem for finitary 2-categories). Let $\mathscr{A}$ and $\mathscr{C}$ be two finitary 2-categories. Then the following assertions are equivalent.

(a) There is a 2-progenerator $\mathscr{P}$ for $\mathscr{C}$ whose endomorphism 2-category is biequivalent to $\mathscr{A}^{\mathrm{p}}$.

(b) The 2-categories $\mathscr{A}$-proj and $\mathscr{C}$-proj are biequivalent.

(c) The 2-categories $\mathscr{A}$-afmod and $\mathscr{C}$-afmod are biequivalent.

5.4. The implication (c) $\Rightarrow(\mathrm{b}) \Rightarrow(\mathrm{a})$. Assume that $\mathscr{A}$-afmod and $\mathscr{C}$-afmod are biequivalent. Since the notion of a projective 2-representation is categorical, it follows that $\mathscr{A}$-proj and $\mathscr{C}$-proj are biequivalent. The image of $\mathscr{P} \mathscr{A}, \mathbb{P}$ under such biequivalence is a 2-progenerator for $\mathscr{C}$.

5.5. The implication $(\mathrm{a}) \Rightarrow(\mathrm{b})$. Denote by $\mathscr{A}$ the endomorphism 2-category of $\mathscr{P}$. Then $\mathscr{\mathscr { A }}^{\mathrm{p}}$ and $\mathscr{A}$ are biequivalent and hence $\mathscr{A}$-afmod and $\mathscr{\mathscr { A }}^{\mathrm{p}}$-afmod are biequivalent by Proposition 3. In particular, $\mathscr{A}$-proj and $\mathscr{\mathscr { A }}^{\mathrm{p}}$-proj are biequivalent by Subsection 5.4. It remains to show that $\mathscr{C}$-proj and $\underline{\mathscr{A}}^{\mathrm{p}}$-proj are biequivalent. We have the obvious 2-functor

$$
\operatorname{Hom}_{\mathscr{C}}\left(\mathscr{P},_{-}\right): \mathscr{C}-\operatorname{proj} \rightarrow \underline{\mathscr{A}}^{\mathrm{p}}-\mathrm{MOD} \text {. }
$$

This 2-functor sends objects of $\mathscr{P}$ to principal, and hence projective, 2-representations of $\mathscr{A}^{\mathrm{p}}$. Let $\mathbf{P}$ be a projective 2-representation of $\mathscr{C}$. By our definition of a 2-progenerator, there is $\mathbf{P}^{\prime}$ in the additive closure of $\mathscr{P}$ and strong transformations $\Psi: \mathbf{P} \rightarrow \mathbf{P}^{\prime}$ and $\Phi: \mathbf{P}^{\prime} \rightarrow \mathbf{P}$ such that $\Phi \Psi$ is isomorphic to the identity on $\mathbf{P}$. Applying $\operatorname{Hom}_{\mathscr{C}}\left(\mathscr{P},{ }_{-}\right)$we get that $\operatorname{Hom}_{\mathscr{C}}\left(\mathscr{P}, \mathbf{P}^{\prime}\right)$ is a direct sum of principal 2-representations of $\mathscr{A}^{\mathrm{p}}$, and $\operatorname{Hom}_{\mathscr{C}}(\mathscr{P}, \mathbf{P})$ comes together with two maps $\operatorname{Hom}_{\mathscr{C}}(\mathscr{P}, \Phi)$ and $\operatorname{Hom}_{\mathscr{C}}(\mathscr{P}, \Psi)$ such that $\operatorname{Hom}_{\mathscr{C}}(\mathscr{P}, \Phi \Psi)$ is isomorphic to the identity on $\operatorname{Hom}_{\mathscr{C}}(\mathscr{P}, \mathbf{P})$. Since $\operatorname{Hom}_{\mathscr{C}}\left(\mathscr{P}, \mathbf{P}^{\prime}\right)$ is a projective 2-representation of $\underline{\mathscr{A}}^{\mathrm{p}}$, so is $\operatorname{Hom}_{\mathscr{C}}(\mathscr{P}, \mathbf{P})$. Therefore

$$
\operatorname{Hom}_{\mathscr{C}}\left(\mathscr{P},_{-}\right): \mathscr{C}-\operatorname{proj} \rightarrow \underline{\mathscr{A}}^{\mathrm{p}}-\text { proj. }
$$

Let $I:=\left\{\dot{i}_{1}, \ldots, \dot{i}_{k}\right\}$ be a cross-section of equivalence classes of objects in $\mathscr{C}$. Let $\mathbb{P}:=\mathbb{P}_{\mathbf{i}_{1}} \oplus \cdots \oplus \mathbb{P}_{\mathbf{i}_{k}}$ and $\mathbf{P}$ a 2 -representation in the additive closure of $\mathscr{P}$ such that there exist strong transformations $\Psi: \mathbb{P} \rightarrow \mathbf{P}$ and $\Phi: \mathbf{P} \rightarrow \mathbb{P}$ with the property that $\Phi \Psi$ is isomorphic to the identity on $\mathbb{P}$. Let $\mathbf{M}$ and $\mathbf{N}$ be two 2-representations of $\mathscr{C}$, let further $\Lambda, \Lambda^{\prime}: \mathbf{M} \rightarrow \mathbf{N}$ be strong transformations and $\eta: \Lambda \rightarrow \Lambda^{\prime}$ a modification. Then the Yoneda Lemma says that $\operatorname{Hom}_{\mathscr{C}}\left(\mathbb{P},{ }_{-}\right)$maps

$$
\mathbf{M} \mapsto \bigoplus_{\mathbf{i} \in \mathrm{I}} \mathbf{M}(\mathbf{i}), \quad \mathbf{N} \mapsto \bigoplus_{\mathbf{i} \in \mathrm{I}} \mathbf{N}(\mathbf{i}), \quad \Lambda \mapsto \bigoplus_{\mathbf{i} \in \mathrm{I}} \Lambda_{\mathbf{i}}, \quad \Lambda^{\prime} \mapsto \bigoplus_{\mathbf{i} \in \mathrm{I}} \Lambda_{\mathbf{i}}^{\prime}, \quad \eta \mapsto \bigoplus_{\mathbf{i} \in \mathrm{I}} \eta_{\mathbf{i}}
$$

Since $\Phi \Psi$ is isomorphic to the identity on $\mathbb{P}$, it follows that composition with $\Phi$ maps $\Lambda, \Lambda^{\prime}$ or $\eta$ to zero if and only if $\Lambda=0, \Lambda^{\prime}=0$ or $\eta=0$, respectively. This implies that the 2 -functor $\operatorname{Hom}_{\mathscr{C}}\left(\mathscr{P},{ }_{-}\right)$from $(12)$ is locally faithful. 
Let $\mathbf{Q}$ denote the multiplicity-free direct sum of all objects in $\mathscr{P}$ up to equivalence (in particular, this sum is finite). By construction, $\mathbb{P}$ is a 2-progenerator for $\mathscr{C}$. Hence there is a direct sum $\mathbf{R}$ of principal 2-representations of $\mathscr{C}$ and strong transformations $\Psi^{\prime}: \mathbf{Q} \rightarrow \mathbf{R}$ and $\Phi^{\prime}: \mathbf{R} \rightarrow \mathbf{Q}$ such that $\Phi^{\prime} \Psi^{\prime}$ is isomorphic to the identity on $\mathbf{Q}$. Since $\mathscr{C}^{\mathrm{op}}$ is biequivalent to $\mathscr{P}_{\mathscr{C}, \mathbb{P}}$, the 2 -functor

$$
\operatorname{Hom}_{\mathscr{C}}\left(\mathscr{P}_{\mathscr{C}, \mathbb{P}},{ }_{-}\right): \mathscr{C} \text {-proj } \rightarrow \operatorname{End}\left(\mathscr{P}_{\mathscr{C}, \mathbb{P}}\right)^{\text {op }} \text {-afmod }
$$

is locally full and dense by Proposition 3. As the additive closures of $\mathscr{P}_{\mathscr{C}, \mathbb{P}}$ and $\mathbb{P}$ coincide, it follows that the 2 -functor

$$
\operatorname{Hom}_{\mathscr{C}}\left(\mathbb{P},_{-}\right): \mathscr{C} \text {-proj } \rightarrow \operatorname{End}(\mathbb{P})^{\mathrm{op}} \text {-afmod }
$$

is locally full and dense. Since $\mathbf{R}$ belongs to the additive closure of $\mathbb{P}$, we get that the 2-functor

$$
\operatorname{Hom}_{\mathscr{C}}\left(\mathbf{R},{ }_{-}\right): \mathscr{C} \text {-proj } \rightarrow \operatorname{End}(\mathbf{R})^{\mathrm{op}} \text {-afmod }
$$

is locally full and dense. Finally, since $\Phi^{\prime} \Psi^{\prime}$ is isomorphic to the identity on $\mathbf{Q}$, composition with $\Psi^{\prime}$ gives that the 2 -functor

$$
\operatorname{Hom}_{\mathscr{C}}\left(\mathbf{Q},_{-}\right): \mathscr{C} \text {-proj } \rightarrow \operatorname{End}(\mathbf{Q})^{\mathrm{op}} \text {-afmod }
$$

is locally full and dense. The latter implies that the 2 -functor $\operatorname{Hom}_{\mathscr{C}}\left(\mathscr{P},{ }_{-}\right)$from (12) is locally full and dense.

It remains to show that the 2 -functor $\operatorname{Hom}_{\mathscr{C}}\left(\mathscr{P},{ }_{-}\right)$from $(12)$ is surjective on equivalence classes of objects. Let now $\mathbf{P}$ be a projective 2-representation of $\mathscr{A}^{\mathrm{p}}$. Without loss of generality we may assume that $\mathscr{A}^{\mathrm{p}}$ is cancellative. By Theorem $15, \mathbf{P}$ is equivalent to a direct sum of 2-representations of the form $\mathbf{P}_{i, \mathrm{E}}^{\mathscr{A}^{\mathrm{op}}}$ for some $i \in \underline{\mathscr{A}}^{\mathrm{p}}$ and $\mathrm{E} \in \mathscr{\mathscr { A }}^{\mathrm{p}}(\mathrm{i}, \mathrm{i})$ such that $\mathrm{E}^{2} \cong \mathrm{E}$. From the definition of $\mathscr{\mathscr { A }}^{\mathrm{p}}$ we have that any such $\mathrm{E}$ comes from an idempotent endomorphism of an object $\mathbf{Q} \in \mathscr{P}$. Since $\underline{\mathscr{A}}^{\mathrm{p}}$ is finitary (in particular, $\mathbb{1}_{\mathrm{i}}$ is indecomposable), $\mathbf{Q}$ has the form $\mathbf{P}_{j, \mathrm{~F}}^{\mathscr{C}}$ for some $j \in \mathscr{C}$ and $F^{2} \cong F \in \mathscr{C}(j, j)$. From Corollary 14(c) it follows that E comes from some $\mathrm{H}^{2} \cong \mathrm{H} \in \mathrm{F} \circ \mathscr{C}(\mathrm{j}, \mathrm{j}) \circ \mathrm{F}$. Consequently, $\operatorname{Hom}_{\mathscr{C}}\left(\mathscr{P}, \mathbf{P}_{j, \mathrm{H}}^{\mathscr{C}}\right) \cong \mathbf{P}_{\mathrm{i}, \mathrm{E}}^{\mathscr{A}^{\circ \mathrm{p}}}$ and hence $\operatorname{Hom}_{\mathscr{C}}\left(\mathscr{P},_{-}\right)$is surjective on equivalence classes of objects.

5.6. The implication $(b) \Rightarrow(c)$. We start with the following observation (recall that the definition of the essential 2-subcategories $t^{A}$ and $\dagger \mathscr{C}$ is in Section 5.2).

Lemma 17. Under assumption (b) the 2-categories $t_{\mathscr{A}}$ and ${ }^{\dagger} \mathscr{C}$ are biequivalent.

Proof. The order $\preceq$ extends in the obvious way to all $\mathbf{P}_{\mathrm{i}, \mathrm{E}}$ in $\mathscr{A}$-proj (and similarly for $\mathscr{C}$-proj). Note that maximal elements with respect to $\preceq$ will be principal. Any biequivalence between $\mathscr{A}$-proj and $\mathscr{C}$-proj maps maximal elements with respect to $\preceq($ for $\mathscr{A})$ to maximal elements with respect to $\preceq$ (for $\mathscr{C}$ ) and hence induces a biequivalence between $\mathfrak{A}^{\prime}$ and ${ }^{\dagger} \mathscr{C}$.

Now from Proposition 3 we have that $t \mathscr{A}$-afmod and $\dagger \mathscr{C}$-afmod are biequivalent. The proof of the implication (b) $\Rightarrow(\mathrm{c})$ is now completed by the following:

Proposition 18. The 2-categories $\mathscr{C}$-afmod and ${ }^{\dagger} \mathscr{C}$-afmod are biequivalent.

Proof. We have the restriction functor

$$
\text { Res : } \mathscr{C} \text {-afmod } \rightarrow^{\dagger} \mathscr{C} \text {-afmod. }
$$


Let $\mathscr{P}$ be a full subcategory of ${ }^{\dagger} \mathscr{C}$-proj consisting of restrictions to ${ }^{\dagger} \mathscr{C}$ of all principal 2 -representations of $\mathscr{C}$. As in the previous subsection we have the obvious 2-functor

$$
\operatorname{Hom}_{+\mathscr{C}}\left(\mathscr{P},_{-}\right):{ }^{\dagger} \mathscr{C} \text {-afmod } \rightarrow \mathscr{C} \text {-afmod. }
$$

Moreover, it is easy to check that $\operatorname{Res} \circ \operatorname{Hom}_{+\mathscr{C}}\left(\mathscr{P},{ }_{-}\right)$is isomorphic to the identity on ${ }^{\dagger} \mathscr{C}$-afmod. In particular, $\operatorname{Hom}_{\dagger \mathscr{C}}\left(\mathscr{P},_{-}\right)$maps non-equivalent 2-representations of $\dagger \mathscr{C}$ to non-equivalent 2-representations of $\mathscr{C}$, moreover, it is locally faithful and locally injective on isomorphism classes of objects.

Let $\mathbf{M}$ and $\mathbf{N}$ be 2-representations of $\mathscr{C}$. Let $\Lambda, \Lambda^{\prime}: \mathbf{M} \rightarrow \mathbf{N}$ be strong transformations and $\alpha: \Lambda \rightarrow \Lambda^{\prime}$ a modification. Let $j$ be an object of $\dagger \mathscr{C}$ and $i$ be an object of $\mathscr{C} \backslash{ }^{\dagger} \mathscr{C}$ such that $i \preceq j$. Following the notation of Subsection 5.2, we have the commutative (up to natural isomorphism) diagram

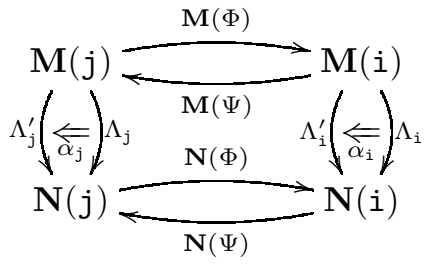

from which we have isomorphisms

$$
\xi_{1}: \Lambda_{i} \rightarrow \mathbf{N}(\Phi) \Lambda_{j} \mathbf{M}(\Psi) \quad \text { and } \quad \xi_{2}: \Lambda_{i}^{\prime} \rightarrow \mathbf{N}(\Phi) \Lambda_{j}^{\prime} \mathbf{M}(\Psi) .
$$

Furthermore, we also have

$$
\alpha_{\mathrm{i}}=\xi_{2}^{-1} \circ_{1}\left(\operatorname{id}_{\mathbf{N}(\Phi)} \circ_{0} \alpha_{j} \circ_{0} \operatorname{id}_{\mathbf{M}(\Psi)}\right) \circ_{1} \xi_{1} .
$$

This proves that Res maps non-equivalent 2-representations of $\mathscr{C}$ to non-equivalent 2-representations of $\dagger \mathscr{C}$, moreover, it also proves that Res is locally faithful and locally injective on isomorphism classes of objects.

Combined with the previous paragraph we have that

- Res and $\operatorname{Hom}_{+\mathscr{C}}\left(\mathscr{P},{ }_{-}\right)$induce mutually inverse bijections between equivalence classes of objects,

- locally they induce mutually inverse bijections between isomorphism classes of 1-morphisms,

- locally they induce injections in both directions on the level of 2-morphisms.

Since 2-morphism spaces are finite dimensional, we obtain that the 2-functors Res and $\operatorname{Hom}_{+\mathscr{C}}\left(\mathscr{P},_{-}\right)$are mutually inverse biequivalences.

\section{EXAMPLES}

6.1. Morita equivalent but not biequivalent finitary 2-categories. Denote by $A$ the path algebra of the quiver $1 \longrightarrow 2$ over $\mathbb{k}$ and let $\mathcal{C}$ be a small category equivalent to $A$-proj. Denote by $\mathrm{F}$ the endofunctor of $\mathcal{C}$ given by tensoring with $A e_{2} \otimes_{\mathrm{k}} e_{2} A$. Clearly, $\mathrm{F}^{2} \cong \mathrm{F}$. Consider the 2-category $\mathscr{C}$ defined as follows:

- $\mathscr{C}$ has one object i (which we identify with $\mathcal{C}$ );

- 1-morphisms in $\mathscr{C}$ are all functors which are isomorphic to a direct sum of copies of $\mathrm{F}$ and the identity functor $\mathbb{1}_{i}=\operatorname{Id}_{\mathcal{C}}$; 
- 2-morphisms in $\mathscr{C}$ are all natural transformations of functors.

Let $\mathscr{A}$ denote the full 2-subcategory of $\mathscr{C}$-proj with objects $\mathbb{P}_{\mathrm{i}}$ and $\mathbf{P}_{\mathrm{i}, \mathrm{F}}$. Set $\mathscr{A}:=\mathscr{A}^{\mathrm{p}}$. Then $\mathscr{A}$ and $\mathscr{C}$ are Morita equivalent by Theorem 16 . On the other hand, $\mathscr{A}$ has two objects which are not equivalent (since the functors representing the actions of $\mathbb{1}_{i}$ and $\mathrm{F}$ on $\mathbf{P}_{i, F}$ are isomorphic while the functors representing the actions of $\mathbb{1}_{i}$ and $\mathrm{F}$ on $\mathbb{P}_{i}$ are not isomorphic). At the same time $\mathscr{C}$ has only one object. Hence $\mathscr{A}$ and $\mathscr{C}$ are not biequivalent.

6.2. Indecomposable non-idempotent 1-morphisms which square to idempotent 1-morphisms. Take $A=\mathbb{k} \oplus \mathbb{k} \oplus \mathbb{k}$ over $\mathbb{k}$, let $\mathcal{D}$ be a small category equivalent to $\mathbb{k}$-mod and $\mathcal{C}:=\mathcal{D} \oplus \mathcal{D} \oplus \mathcal{D}$ (which is equivalent to $A$-proj $=A$-mod). Denote by $\mathrm{E}$ the identity functor on $\mathcal{D}$ and let $\mathrm{F}$ and $\mathrm{K}$ be the endofunctors of $\mathcal{C}$ given by the matrices

$$
\left(\begin{array}{ccc}
0 & \mathrm{E} & 0 \\
0 & \mathrm{E} & \mathrm{E} \\
0 & 0 & 0
\end{array}\right) \text { and }\left(\begin{array}{ccc}
0 & 0 & \mathrm{E} \\
0 & 0 & 0 \\
0 & 0 & 0
\end{array}\right),
$$

respectively. We have $\mathrm{K}^{2}=\mathrm{KF}=\mathrm{FK}=0$ and $\mathrm{F}^{2} \cong \mathrm{F} \oplus \mathrm{K}$, from which it follows that $(\mathrm{F} \oplus \mathrm{K})^{2} \cong \mathrm{F} \oplus \mathrm{K}$. Consider the 2-category $\mathscr{C}$ defined as follows:

- $\mathscr{C}$ has one object i (which we identify with $\mathcal{C}$ );

- 1-morphisms in $\mathscr{C}$ are all functors which are isomorphic to a direct sum of copies of $\mathrm{F}, \mathrm{K}$ and the identity functor $\mathbb{1}_{\mathrm{i}}=\mathrm{Id}_{\mathcal{C}}$;

- 2-morphisms in $\mathscr{C}$ are given by scalar multiples of the identity natural transformations on $\mathrm{F}, \mathrm{K}$ and $\mathbb{1}_{\mathfrak{i}}$, extended additively to their direct sums.

The 1-morphism $\mathrm{F}$ in $\mathscr{C}$ is an indecomposable non-idempotent 1-morphism which squares to an idempotent (but decomposable) 1-morphism.

6.3. Morita equivalence classes for 2-categories of projective functors for finite dimensional algebras. Let $A$ be a finite dimensional $\mathbb{k}$-algebra. Assume that $A \cong A_{1} \oplus A_{2} \oplus \cdots \oplus A_{k}$ with $A_{1}, A_{2}, \ldots, A_{k}$ connected (that is indecomposable as algebras). Denote by $\mathscr{C}_{A}$ the 2-category defined as follows (compare [MM1, 7.3]):

- objects are $1, \ldots, \mathrm{k}$ where we identify $\mathrm{i}$ with some small category equivalent to $A_{i}$-proj;

- 1-morphisms are all additive functors from $A_{i}$-proj to $A_{j}$-proj isomorphic to direct sums of functors realized as tensoring with either projective $A_{j}-A_{i^{-}}$ bimodules or, additionally, with the bimodule $A_{i}$ in case $i=j$;

- 2-morphisms are natural transformations of functors.

Note that, up to biequivalence, $\mathscr{C}_{A}$ does not depend on the choice of small categories equivalent to $A_{i}$-proj for $i=1,2, \ldots, k$. The 2-category $\mathscr{C}_{A}$ is of particular interest, as in [MM3, Theorem 13] it was shown that fiat 2-categories, which are "simple" in a certain sense, are constructed from these.

For $B \cong B_{1} \oplus B_{2} \oplus \cdots \oplus B_{m}$ with $B_{1}, B_{2}, \ldots, B_{m}$ connected, write $A \sim B$ provided that the following two conditions are satisfied: 
- $m=k+1, A_{1} \cong B_{1}, A_{2} \cong B_{2}, \ldots, A_{k} \cong B_{k}$ and $B_{m} \cong \mathbb{k}$

- there are idempotents $e, e^{\prime} \in A$ such that $\operatorname{dim} e A e^{\prime}=1$.

Denote by $\approx$ the minimal equivalence relation (on the class of all finite dimensional $\mathbb{k}$-algebra) containing both $\sim$ and the classical Morita equivalence relation for finite dimensional algebras.

Theorem 19. Let $A$ and $B$ be two finite dimensional $\mathbb{k}$-algebras. Then $\mathscr{C}_{A}$ and $\mathscr{C}_{B}$ are Morita equivalent if and only if $A \approx B$.

Proof. We first note that the 2-category $\mathscr{C}_{A}$ is, clearly, independent of the choice of $A$ within its Morita equivalence class. Hence, to prove sufficiency it is enough to show that $A \sim B$ implies Morita equivalence of $\mathscr{C}_{A}$ and $\mathscr{C}_{B}$. Let $\mathbb{P}_{\mathrm{m}}^{\mathscr{C}_{B}}$ be the principal 2-representation of $B$ associated to $B_{m}$. We identify $A$ with the subalgebra $B_{1} \oplus \cdots \oplus B_{k}$ of $B$. Let $x$ denote the identity in $B_{m}$. Then $B x \otimes_{\mathbb{k}} e A$ and $A e^{\prime} \otimes_{\mathbb{k}} x B$ are 1-morphisms in $\mathscr{C}_{B}$. As $\operatorname{dim} e A e^{\prime}=1$, we have

$$
B x \otimes_{\mathbb{k}} e A \otimes_{B} A e^{\prime} \otimes_{\mathbb{k}} x B \cong B x \otimes_{\mathbb{k}} x B^{\oplus \operatorname{dim} e A e^{\prime}} \cong B_{m} .
$$

This implies that $\mathbb{P}_{\mathrm{m}}^{\mathscr{C}_{B}}$ is a retract of $\mathbb{P}_{1}^{\mathscr{C}_{B}} \oplus \cdots \oplus \mathbb{P}_{\mathrm{k}}^{\mathscr{C}_{B}}$. By our choice of $A$ we have that the endomorphism 2-category of $\mathbb{P}_{1}^{\mathscr{C} B}, \mathbb{P}_{2}^{\mathscr{C}_{B}}, \ldots, \mathbb{P}_{\mathrm{k}}^{\mathscr{C}_{B}}$ is biequivalent to the endomorphism 2-category of $\mathbb{P}_{1}^{\mathscr{C}_{A}}, \mathbb{P}_{2}^{\mathscr{C}_{A}}, \ldots, \mathbb{P}_{\mathrm{k}}^{\mathscr{C}_{A}}$. The claim follows.

To prove necessity, let us analyze idempotent 1-morphisms in $\mathscr{C}_{A}$. Let $e, e^{\prime}$ be primitive idempotents in $A$ and $A e^{\prime} \otimes_{\mathbb{k}} e A$ the corresponding projective bimodule. We have

$$
A e^{\prime} \otimes_{\mathbb{k}} e A \otimes_{A} A e^{\prime} \otimes_{\mathbb{k}} e A \cong A e^{\prime} \otimes_{\mathbb{k}} e A^{\oplus \operatorname{dim} e A e^{\prime}}
$$

and hence

$$
\begin{aligned}
\left(A e^{\prime} \otimes_{\mathbb{k}} e A \otimes_{A} A e^{\prime} \otimes_{\mathbb{k}} e A\right) \otimes_{A}\left(A e^{\prime} \otimes_{\mathbb{k}} e A \otimes_{A} A e^{\prime} \otimes_{\mathbb{k}} e A\right) & \\
& \cong\left(A e^{\prime} \otimes_{\mathbb{k}} e A \otimes_{A} A e^{\prime} \otimes_{\mathbb{k}} e A\right)^{\oplus\left(\operatorname{dim} e A e^{\prime}\right)^{2}} .
\end{aligned}
$$

This implies that $A e^{\prime} \otimes_{\mathbb{k}} e A \otimes_{A} A e^{\prime} \otimes_{\mathbb{k}} e A$ is idempotent if and only if $\operatorname{dim} e A e^{\prime}=1$. Note that, by (13), the latter is equivalent to $A e^{\prime} \otimes_{\mathbb{k}} e A$ being idempotent. By Theorem 16, the Morita equivalence class of a finitary 2-category is obtained by adding or removing retracts of indecomposable principal 2-representations. Let $\mathbf{P}$ be a retract corresponding to an idempotent $A e^{\prime} \otimes_{\mathbb{k}} e A$. Since $A e^{\prime} \otimes_{\mathbb{k}} e A$ is idempotent, it factors through $\mathbb{k}$-proj. This implies that the endomorphism 2category of $\mathbb{P}_{1}, \mathbb{P}_{2}, \ldots, \mathbb{P}_{\mathrm{k}}, \mathbf{P}$ is biequivalent to $\mathscr{C}_{B}$ where $B=A \oplus \mathbb{k}$. This completes the proof.

6.4. 2-categories of Soergel bimodules. Let $(W, S)$ be a finite Coxeter system and $\mathbf{C}_{W}$ the corresponding coinvariant algebra (see [Wi] for details). Let $\mathscr{S}_{(W, S)}$ be a 2-category of Soergel $\mathbf{C}_{W}-\mathbf{C}_{W}$-bimodules for $(W, S)$ as defined in [MM1, Subsection 7.1] or [MM2, Example 3] (in those papers $W$ is assumed to be a Weyl group, however, our more general assumption works just fine, see [EW, Wi]). The 2-category $\mathscr{S}_{(W, S)}$ is usually described using its defining 2-representation:

- $\mathscr{S}_{(W, S)}$ has one object which is identified with some small category $\mathcal{A}$ equivalent to $\mathbf{C}_{W}$-mod;

- 1-morphisms of $\mathscr{S}_{(W, S)}$ are endofunctors of $\mathcal{A}$ isomorphic to direct sums of endofunctors given by tensoring with Soergel bimodules; 
- 2-morphisms of $\mathscr{S}_{(W, S)}$ are natural transformations of functors.

Clearly, up to biequivalence, $\mathscr{S}_{(W, S)}$ does not depend on the choice of $\mathcal{A}$.

Proposition 20. Let $(W, S)$ and $\left(W^{\prime}, S^{\prime}\right)$ be finite Coxeter systems. Then the 2 -categories $\mathscr{S}_{(W, S)}$ and $\mathscr{S}_{\left(W^{\prime}, S^{\prime}\right)}$ are Morita equivalent if and only if $(W, S)$ and $\left(W^{\prime}, S^{\prime}\right)$ are isomorphic.

Proof. The "if" part is obvious. The "only if" part follows from Theorem 16 and the following two observations.

Observation 1. The 2-categories $\mathscr{S}_{(W, S)}$ and $\mathscr{S}_{\left(W^{\prime}, S^{\prime}\right)}$ are biequivalent if and only if $(W, S)$ and $\left(W^{\prime}, S^{\prime}\right)$ are isomorphic. The "if" part is again obvious. To prove the "only if" part, note that any biequivalence between $\mathscr{S}_{(W, S)}$ and $\mathscr{S}_{\left(W^{\prime}, S^{\prime}\right)}$ induces an isomorphism between the 2-endomorphism algebras of the identity 1-morphisms in $\mathscr{S}_{(W, S)}$ and $\mathscr{S}_{\left(W^{\prime}, S^{\prime}\right)}$. By definition, these endomorphism algebras are isomorphic to $\mathbf{C}_{W}$ and $\mathbf{C}_{W^{\prime}}$, respectively. Finally, it is easy to check that $\mathbf{C}_{W} \cong \mathbf{C}_{W^{\prime}}$ if and only if $(W, S) \cong\left(W^{\prime}, S^{\prime}\right)$.

Observation 2. The only weakly idempotent 1-morphism in $\mathscr{S}_{(W, S)}$ is the identity 1morphism. Tensoring with a Soergel bimodule is a non-zero and exact endofunctor of $\mathcal{A}$ and the latter category has only one simple object up to isomorphism (call it $L$ ). Let $\mathrm{G}$ be an indecomposable 1-morphism in $\mathscr{S}_{(W, S)}$ such that $\mathrm{F}:=\mathrm{G} \circ \mathrm{G} \cong \mathrm{G} \oplus \mathrm{Q}$ is weakly idempotent. Let $m$ be the length of $\mathrm{F}(L) \cong \mathrm{F}^{2}(L)$. Then $m=m^{2}$ and hence $m=1$ (since $\mathrm{G}$ is nonzero). It follows that $\mathrm{Q}=0$ and thus $\mathrm{F}=\mathrm{G}$. In particular, $\mathrm{F}$ is indecomposable and hence corresponds to some element $w \in W$ and, moreover, $\mathrm{F}(L) \cong L$. In the natural graded picture $\mathrm{F}(L)$ is a graded self-dual vector space with non-zero components in degrees \pm the length of $w$. Therefore $\mathrm{F}(L) \cong L$ implies that the length of $w$ equals zero and hence $w$ must coincide with the identity element. Therefore $\mathrm{F}$ is isomorphic to the identity 1-morphism. This completes the proof.

\section{REFERENCES}

[Ar] S. Ariki; On the decomposition numbers of the Hecke algebra of $G(m, 1, n)$. J. Math. Kyoto Univ. 36 (1996), no. 4, 789-808.

[BD] F. Borceux, D. Dejean; Cauchy completion in category theory. Cahiers Topologie Géom. Différentielle Catég. 27 (1986), no. 2, 133-146.

[BV] G. Böhm, J. Vercruysse; Morita theory for comodules over corings. Comm. Algebra 37 (2009), no. 9, 3207-3247.

[CR] J. Chuang, R. Rouquier; Derived equivalences for symmetric groups and $\mathfrak{s l}_{2}$-categorification. Ann. of Math. (2) 167 (2008), no. 1, 245-298.

[EW] B. Elias, G. Williamson; The Hodge theory of Soergel bimodules. Preprint arXiv:1212.0791.

[Fl] P. Flor; On groups of non-negative matrices. Compositio Math. 21 (1969), 376-382.

[Gr] I. Grojnowski; Affine $\mathfrak{s l}_{p}$ controls the representation theory of the symmetric group and related Hecke algebras. Preprint arXiv:math/9907129.

[Ke] B. Keller; On differential graded categories. International Congress of Mathematicians. Vol. II, 151-190, Eur. Math. Soc., Zürich, 2006.

[Kh] M. Khovanov; A categorification of the Jones polynomial. Duke Math. J. 101 (2000), no. $3,359-426$.

[Kn] U. Knauer; Projectivity of acts and Morita equivalence of monoids. Semigroup Forum 3 (1971/1972) no. 4, 359-370.

[LLT] A. Lascoux, B. Leclerc, J.-Y. Thibon; Hecke algebras at roots of unity and crystal bases of quantum affine algebras. Comm. Math. Phys. 181 (1996), no. 1, 205-263.

[Le] T. Leinster; Basic bicategories. Preprint arXiv:math/9810017. 
[MM1] V. Mazorchuk, V. Miemietz; Cell 2-representations of finitary 2-categories; Compositio Math. 147 (2011), 1519-1545.

[MM2] V. Mazorchuk, V. Miemietz; Additive versus abelian 2-representations of fiat 2-categories. Preprint arxiv:1112.4949.

[MM3] V. Mazorchuk, V. Miemietz; Endomorphisms of cell 2-representations. Preprint arXiv:1207.6236.

[MOS] V. Mazorchuk, S. Ovsienko, C. Stroppel; Quadratic duals, Koszul dual functors, and applications. Trans. Amer. Math. Soc. 361 (2009), no. 3, 1129-1172.

[Mo] K. Morita; Duality for modules and its applications to the theory of rings with minimum condition. Sci. Rep. Tokyo Kyoiku Daigaku Sect. A 6 (1958) 83-142.

[Ri] J. Rickard; Morita theory for derived categories. J. London Math. Soc. (2) 39 (1989), no. 3, 436-456.

[St] C. Stroppel; Categorification of the Temperley-Lieb category, tangles, and cobordisms via projective functors. Duke Math. J. 126 (2005), no. 3, 547-596.

[To] B. Toën; The homotopy theory of dg-categories and derived Morita theory. Invent. Math. 167 (2007), no. 3, 615-667.

[Wi] G. Williamson; Singular Soergel bimodules. Int. Math. Res. Not. 2011, no. 20, 45554632 .

Volodymyr Mazorchuk, Department of Mathematics, Uppsala University, Box 480, 751 06, Uppsala, SWEDEN, mazor@math.uu.se; http://www.math.uu.se/mazor/.

Vanessa Miemietz, School of Mathematics, University of East Anglia, Norwich, UK, NR4 7TJ, v.miemietz@uea.ac.uk; http://www.uea.ac.uk/'byr09xgu/. 